University of Tennessee Health Science Center

UTHSC Digital Commons

\title{
Synthesis and Characterization of Methacrylated Hyaluronan- Based Hydrogels for Tissue Engineering
}

Keegan Bradley Compton

University of Tennessee Health Science Center

Follow this and additional works at: https://dc.uthsc.edu/dissertations

Part of the Medical Biotechnology Commons, and the Other Medical Sciences Commons

\section{Recommended Citation}

Compton, Keegan Bradley , "Synthesis and Characterization of Methacrylated Hyaluronan-Based Hydrogels for Tissue Engineering" (2014). Theses and Dissertations (ETD). Paper 47. http://dx.doi.org/ 10.21007/etd.cghs.2014.0056.

This Thesis is brought to you for free and open access by the College of Graduate Health Sciences at UTHSC Digital Commons. It has been accepted for inclusion in Theses and Dissertations (ETD) by an authorized administrator of UTHSC Digital Commons. For more information, please contact jwelch30@uthsc.edu. 


\title{
Synthesis and Characterization of Methacrylated Hyaluronan-Based Hydrogels for Tissue Engineering
}

\begin{abstract}
Polymers have revolutionized the field of tissue engineering due to the countless possibilities of scaffolds that can be constructed from such a material. Through conjugation of other functional groups onto a polymer backbone, the mechanical properties of polymers can be controlled and has the ability to span a wide range of strengths, allowing for application in multiple tissue environments. One such polymer, hyaluronic acid (HA), is a natural polysaccharide found most abundantly in the extracellular matrix (ECM) of connective tissues with structural, lubricating, and wound healing functions in the human body; for this reason, $\mathrm{HA}$ has great potential for utilization in tissue engineering application. To strengthen the mechanical properties of HA, we conjugated 2-aminoethyl methacrylate (AEMA) to the carboxyl group of $\mathrm{HA}$ polymer in various degrees of substitution. In addition, different molecular weights of HA were used during synthesis to analyze molecular weight as a factor for tunable mechanical properties. The final product HA-AEMA, with use of photoinitiator Irgacure 2959, forms a hydrogel via UV polymerization following dissolution of macromer into photoinitiator solution. Both the rheological and mechanical properties of various macromer/photoinitiator solutions and hydrogels were measured, respectively, in regards to viscosity, solubility, shear modulus, and compression strength. To test HA-AEMA potential for use of tissue engineering applications involving stem cells, human dental pulp stem cells (DPSCs) were exposed to both HA-AEMA macromer and hydrogels for cytotoxicity and encapsulation studies by MTT using mitochondrial based assays and fluorescent viable cell staining respectively. Results of our experiments indicate successful synthesis of HA-AEMA macromer of various molecular weights of HA as well as degree of substitution of AEMA. As the molecular weight of HA and/or the degree of substitution of AEMA increased, the viscosity, swelling ratio, shear storage modulus, and compressive strength increased; specifically for storage modulus, more significant increases were observed as MW of HA was increased from $16 \mathrm{kDa}$ to $66 \mathrm{kDa}$ compared to increases from $66 \mathrm{kDa}$ to $270 \mathrm{kDa}$. As the hydrogels swelled over time in phosphate buffer solution (PBS), the compressive strength of hydrogels diminished by a factor of 3 after 24 hours of swelling time and a factor of 2 from 1 day to 3 days swelling time, most likely due to hydrolysis of the ester bond along the HA chain. Results of the cytotoxicity studies indicate DPSCs survive exposure to HA-AEMA macromer as well as encapsulation inside hydrogels formed following the standard UV polymerization protocol. Based on these results, we have concluded that HAbased hydrogels have the potential to be used in various tissue engineering applications due to its tunable properties and biocompatibility to DPSCs
\end{abstract}

\section{Document Type}

Thesis

\section{Degree Name}

Master of Science (MS)

\section{Program}

Biomedical Engineering

\section{Research Advisor}

Tao L. Lowe, Ph.D.

\section{Keywords}

2-aminoethyl methacrylate dental pulp stem cells hyaluronic acid hydrogels tissue engineering UV polymerization 


\section{Subject Categories}

Medical Biotechnology | Medicine and Health Sciences | Other Medical Sciences 


\title{
Synthesis and Characterization of Methacrylated Hyaluronan-Based Hydrogels for Tissue Engineering
}

\author{
A Thesis \\ Presented for \\ The Graduate Studies Council \\ The University of Tennessee \\ Health Science Center
}

\begin{abstract}
In Partial Fulfillment
Of the Requirements for the Degree

Master of Science

In the Joint Graduate Program in Biomedical Engineering and Imaging

From The University of Tennessee

and

The University of Memphis
\end{abstract}

By

Keegan Bradley Compton

December 2014 
Copyright (C) 2014 by Keegan Bradley Compton.

All rights reserved 


\section{DEDICATION}

I would like to dedicate this thesis to my family for all of their love and support, and to Kayla, who I love very much. Thank you. 


\section{ACKNOWLEDGEMENTS}

I would like to thank everyone who helped me complete this thesis work. First, thank you to Dr. Tao Lowe for all her advice and guidance. I would also like to thank my committee members, Dr. Richard Smith, Dr. George Huang, and Dr. Joel Bumgardner for their assistance. I would also like to thank Linfeng Wu, Xiaoxun Li, Dileep Janagam, Sibo Jiang, and Ikbale Ayachi for their help. 


\begin{abstract}
Polymers have revolutionized the field of tissue engineering due to the countless possibilities of scaffolds that can be constructed from such a material. Through conjugation of other functional groups onto a polymer backbone, the mechanical properties of polymers can be controlled and has the ability to span a wide range of strengths, allowing for application in multiple tissue environments. One such polymer, hyaluronic acid (HA), is a natural polysaccharide found most abundantly in the extracellular matrix (ECM) of connective tissues with structural, lubricating, and wound healing functions in the human body; for this reason, HA has great potential for utilization in tissue engineering application. To strengthen the mechanical properties of HA, we conjugated 2-aminoethyl methacrylate (AEMA) to the carboxyl group of HA polymer in various degrees of substitution. In addition, different molecular weights of HA were used during synthesis to analyze molecular weight as a factor for tunable mechanical properties. The final product HA-AEMA, with use of photoinitiator Irgacure 2959 , forms a hydrogel via UV polymerization following dissolution of macromer into photoinitiator solution. Both the rheological and mechanical properties of various macromer/photoinitiator solutions and hydrogels were measured, respectively, in regards to viscosity, solubility, shear modulus, and compression strength. To test HA-AEMA potential for use of tissue engineering applications involving stem cells, human dental pulp stem cells (DPSCs) were exposed to both HA-AEMA macromer and hydrogels for cytotoxicity and encapsulation studies by MTT using mitochondrial based assays and fluorescent viable cell staining respectively. Results of our experiments indicate successful synthesis of HA-AEMA macromer of various molecular weights of HA as well as degree of substitution of AEMA. As the molecular weight of HA and/or the degree of substitution of AEMA increased, the viscosity, swelling ratio, shear storage modulus, and compressive strength increased; specifically for storage modulus, more significant increases were observed as MW of HA was increased from $16 \mathrm{kDa}$ to $66 \mathrm{kDa}$ compared to increases from $66 \mathrm{kDa}$ to $270 \mathrm{kDa}$. As the hydrogels swelled over time in phosphate buffer solution (PBS), the compressive strength of hydrogels diminished by a factor of 3 after 24 hours of swelling time and a factor of 2 from 1 day to 3 days swelling time, most likely due to hydrolysis of the ester bond along the HA chain. Results of the cytotoxicity studies indicate DPSCs survive exposure to HA-AEMA macromer as well as encapsulation inside hydrogels formed following the standard UV polymerization protocol. Based on these results, we have concluded that HA-based hydrogels have the potential to be used in various tissue engineering applications due to its tunable properties and biocompatibility to DPSCs.
\end{abstract}




\section{TABLE OF CONTENTS}

CHAPTER 1. INTRODUCTION .................................................................................

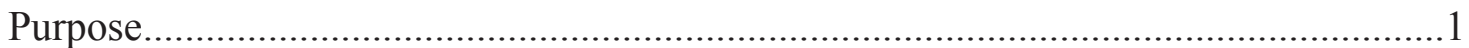

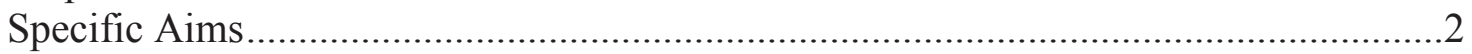

CHAPTER 2. LITERATURE REVIEW ...............................................................4

Current Definition and Treatment of Tissue Injury .............................................

At-Home and Surgical Treatment of Tissue Injury ...............................................

Biomaterials Used in the Treatment of Tissue Injury ..............................................4

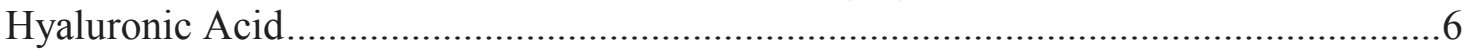

Role and Structure in the Body ..............................................................................6

Commercial Uses of Hyaluronic Acid as a Scaffold ............................................. 7

Hyaluronic Acid Structure Modification for Scaffold Use .................................... 7

HA-Based Hydrogels for Tissue Engineering Purposes ............................................8

CHAPTER 3. MATERIALS AND METHODS...................................................10

Synthesize and Characterize HA-AEMA Macromer................................................ 10

Synthesis Process of HA-AEMA Macromer ........................................................ 10

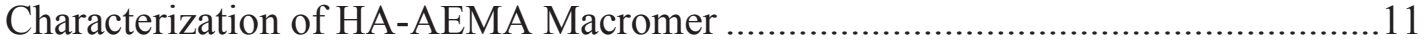

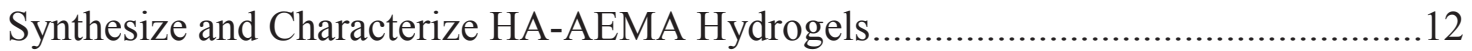

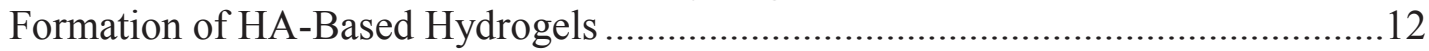

Investigation of Mechanical and Rheological Properties of HA-Based Hydrogels...12

Study Cytotoxicity and Encapsulation Capabilities of HA-AEMA Macromers and

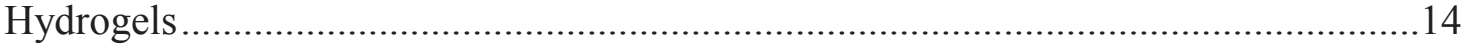

Cytotoxicity of HA-AEMA Macromer to DPSCs .................................................. 14

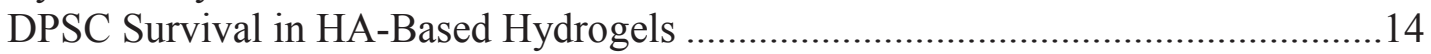

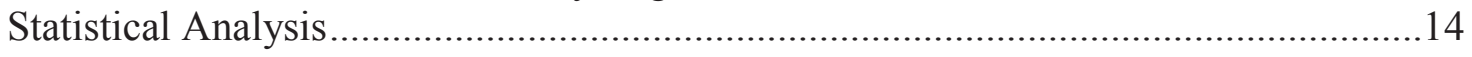

CHAPTER 4. RESULTS.......................................................................................................15

Synthesis and Characterization of HA-AEMA Macromer ...................................... 15

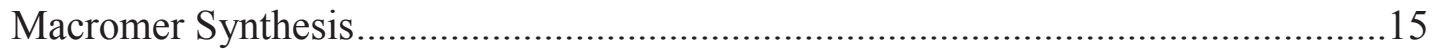

HA-AEMA Macromer Characterization........................................................... 17

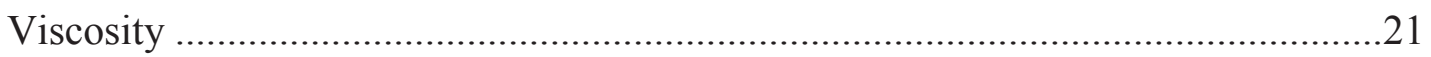

Synthesis and Characterization of HA-AEMA Hydrogels .....................................21

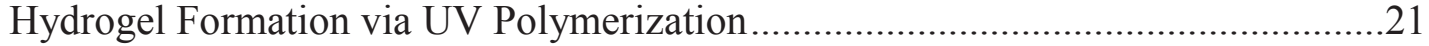

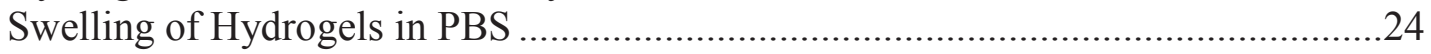

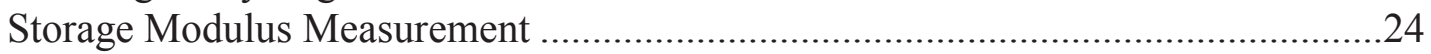

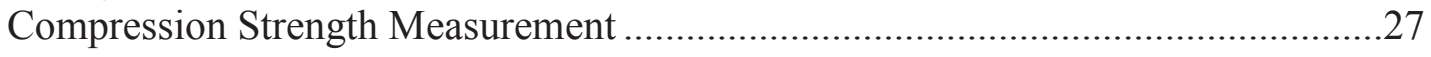

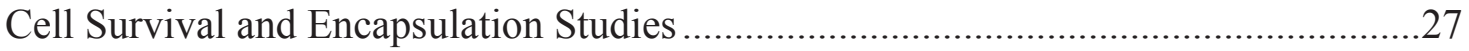

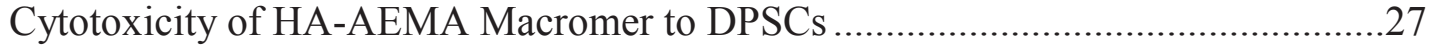

DPSC Encapsulation in Hydrogel.................................................................... 30

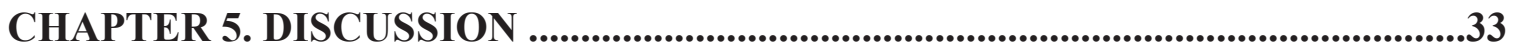


CHAPTER 6. CONCLUSION ..............................................................................35

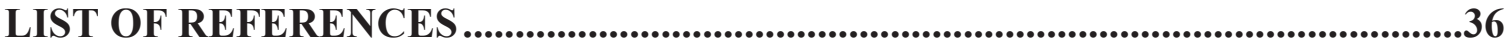

VITA 


\section{LIST OF TABLES}

Table 4-1. Solubility test of HA-AEMA macromers in PBS ....................................20

Table 4-2. Hydrogel formation of HA-AEMA macromers via UV polymerization......23 


\section{LIST OF FIGURES}

Figure 4-1. ${ }^{1}$ NMR of HA-TBA following ion exchange process of synthesis...............16

Figure 4-2. Vacuum drying precipitate affects end product of synthesis. ......................18

Figure 4-3. NMR graph of HA-AEMA-18k-25 for structure characterization. ..............19

Figure 4-4. Viscosity of HA-AEMA-16k-25, HA-AEMA-66k-25, and HA-AEMA-

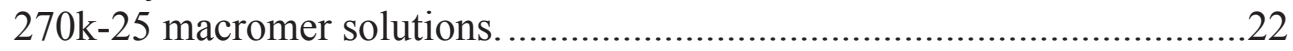

Figure 4-5. Difference in swollen hydrogels based on AEMA degree of substitution....25

Figure 4-6. Storage modulus comparisons based on MW of HA, AEMA degree of

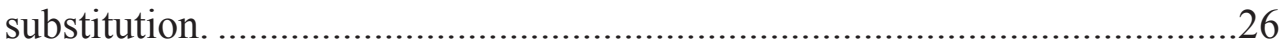

Figure 4-7. Profile of stress/strain curve for HA-AEMA hydrogel compression............28

Figure 4-8. Compressive moduli of HA-AEMA-18k-25 hydrogels over time. .............29

Figure 4-9. Cytotoxicity of HA-AEMA macromer to dental pulp stem cells................31

Figure 4-10. LIVE cell staining of encapsulated dental pulp stem cells in HA-AEMA

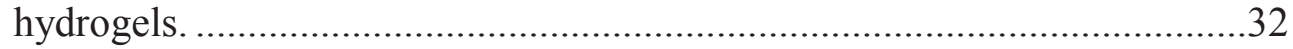




\section{LIST OF ABBREVIATIONS}

AEMA 2-Aminoethyl Methacrylate

DPSC Dental Pulp Stem Cell

ECM Extracellular Matrix

HA Hyaluronic Acid

NMR Nuclear Magnetic Resonance

TBA Tetrabutylammonium 


\section{CHAPTER 1. INTRODUCTION}

\section{Purpose}

In the past few decades, tissue engineering has grown from a subset of biomaterials into a self-standing field. Tissue engineering focuses on the repair of damaged tissue and maintenance of the wound healing process to minimize scar tissue formation through the development of biocompatible technologies and materials in vitro (Chen, Liang et al. 2013). The rapid expansion of the tissue engineering field has occurred for a number of reasons, one of the most important being the innovation of polymers for use in medical applications (Keane and Badylak 2014). Prior to polymers, biomaterials were limited to ceramics and metals; these biomaterials generally have mechanical properties that differ greatly from native soft tissues, and this limited their major use to orthopedic implants (Chen, Liang et al. 2013). Because of this, treatment of severe injuries to soft tissue was only possible through invasive surgery and focused primarily on reducing the pain and side effects of the patient over returning the damaged tissue to full function. Surgical treatment of tissue damaged beyond repair often includes use of autografts, tissue that comes from a separate site on the patient. When damaged tissue was beyond repair, autografts are often used. However, use of autografts requires additional surgeries for care of the donor site. Allografts may be employed to avoid sacrificing skin from a patient donor site, but use of allografts comes with the risk of tissue rejection as well as disease transmission. To avoid these risks and while aiding in the healing process to return tissue to full function, biomaterials need to be utilized, and polymers are currently being investigated to fill this need. Polymers have great potential in giving more treatment options to physicians, reducing invasive surgery treatment by autografts and allografts, and healing damaged tissue more effectively.

Polymers have a vast range of mechanical properties that allows them to be used in a wide variety of tissue engineering applications. This is advantageous since human tissue ranges greatly; for example, stresses present in the body can range from 0.1 to 80 $\mathrm{kPa}$, seen in brain and bone environments, respectively (Li, Katsanevakis et al. 2012, Guvendiren and Burdick 2013). Although some metal and ceramic biomaterials are made to be bioactive, such polymers can be used for treatment in a greater number of tissues while maintaining biocompatibility based on their wider range of possible mechanical properties. Lastly, polymers can have controlled degradation: some polymer biomaterials can be made to degrade by the body's natural processes, such as breakdown by hydrolysis and enzymes, eliminating the need for invasive surgery for removal (Zhao, Tan et al. 2013). In order to optimize these advantages, certain traits of these polymers are controlled, for example the molecular weight, cross-linking density, monomer unit selection, and method of polymerization (Guvendiren and Burdick 2013). By using a combination of different polymers and stem cells, tissue engineering application can extend to many different tissue types, including bone, cartilage, neural, cardiac, ocular, and other tissue types (Zhao, Tan et al. 2013) By controlling polymer traits, countless polymers have potential to be used in medical applications, and new polymers are being discovered and studied to widen the foundation of tissue engineering. 
One particular polymer of interest in current research is hyaluronic acid (HA), a naturally occurring polysaccharide commonly found in the extracellular matrix of connective tissues. HA has been studied extensively for tissue engineering applications because of its wound healing capabilities and structural support roles in the body. In order to maximize the utilization of HA beyond the scope of that found naturally in the body, conjugation of a moiety to an HA backbone while controlling the molecular weight of HA allows for the biopolymer to span a wide range of mechanical properties for potential use in multiple tissue engineering applications (Price, Berry et al. 2007).

One modification to HA that is used to strengthen the mechanical properties of the polymer is conjugation of a methacrylate group; this conjugation allows the possibility of cross-linking and formation of a polymer network that is stronger than HA alone. The resulting polymer, in the presence of a photoinitiator, can be cross-linked via UV polymerization, and this forms a hydrophilic scaffold called a hydrogel (Bae, 2011; Schante, 2011; Nguyen, 2014). For our work, we conjugated HA with 2-aminoethyl methacrylate (AEMA), forming HA-AEMA macromer. With massive swelling capabilities, the ability to encapsulate cells and other factors, biodegradability, and a backbone known for having wound healing and structural roles in the body, HA-AEMA hydrogels have potential to be effective tissue engineering scaffolds. To investigate the extent of scaffold possibilities, we synthesized HA-AEMA macromers of various HA molecular weights and cross-linking densities of AEMA and analyzed their rheological and mechanical properties. Furthermore, we exposed dental pulp stem cells (DPSCs), to both HA-AEMA macromer and hydrogel to test for cytotoxicity. The purpose of this work is to evaluate the range of mechanical properties possible for HA-based hydrogels as well as their cytotoxicity to DPSCs to determine the extent of their potential utility in various tissue engineering applications.

\section{Specific Aims}

The long term goal of this project is to develop the HA-AEMA hydrogel whose mechanical properties are optimized for successful growth, encapsulation, and differentiation of stem cells for tissue engineering applications. This hydrogel would have tunable mechanical properties in order to match those of native tissue, and it would degrade over time so the encapsulated cells would have space and time to grow, differentiate, and aid in the healing and restructuring of damaged tissue. A desired result of this optimized scaffold system would be higher occurrence rates of recovery of functionality.

In order to achieve this goal, we must first identify factors that control the mechanical properties, and make sure the hydrogel system is biocompatible with cells. The objective of this thesis was to synthesize HA-AEMA based hydrogels of $18 \mathrm{kDa}$ and $270 \mathrm{kDa} H A$ molecular weights and characterize them along with other previously synthesized HA-AEMA hydrogels. Another objective was to show that these hydrogels

are able to successfully encapsulate dental pulp stem cells. The main hypothesis of this 
thesis is HA-based hydrogels can be developed with tunable mechanical properties and can successfully encapsulate DPSC cells as representative stem cell type in order to validate potential use for tissue engineering applications. This hypothesis was tested according to the following three specific aims:

1) Synthesize and characterize HA macromer.

Through ion exchange, AEMA conjugation, and dialysis, HA was converted from a sodium salt configuration to a methacrylated form, specifically conjugated with 2-aminoethyl methacrylate. Two macromers that vary in the molecular weight of the HA component will be synthesized. Verification of successful synthesis will be done by ${ }^{1} \mathrm{H}$ NMR. In order to characterize the HA-AEMA macromers, we will check which macromers are soluble in solution as well as measure their viscosity in solution.

2) Synthesize and characterize the mechanical properties of HA-based hydrogels. Synthesis of HA-based hydrogels was done via UV polymerization, and both newly-synthesized and previously-synthesized macromers were tested to see which resulted in successful formation of a hydrogel. Those hydrogels that were successfully synthesized were characterized by measuring their viscoelastic and mechanical properties, including storage and loss moduli, swelling ratio, and compressive strength. In addition, the shape of hydrogels was characterized over various swelling times.

3) Study cytotoxicity and encapsulation capabilities of HA macromer and hydrogels. DPSCs were cultured in flasks until confluent. Cytotoxicity of DPSCs to HA macromer was tested at various concentrations of macromer in cell growth medium by MTT assay. Encapsulation capabilities were studied by mixing suspended stem cells to the macromer/photoinitiator solution and exposing the mixture to UV during the polymerization process. After 24 hours, hydrogels were stained for live cells to visualize successful encapsulation and survival of stem cells during the hydrogel formation process. Effects of UV exposure to encapsulated cells were not evaluated during this study. 


\section{CHAPTER 2. LITERATURE REVIEW}

\section{Current Definition and Treatment of Tissue Injury}

Injury to tissue occurs in a variety of ways, such as injury from sports and exercise, falls, car accidents, or other high impact traumatic events. Whatever the cause of the injury, the effect is a mechanical force that extends beyond the strength of the tissue itself. Examples of such injuries include sprains and strains of ligaments and tendons, muscle fatigue, tissue tearing, crush injury of nerve tissue, lacerations, and more. Tissue injury can be a painful event that is accompanied with swelling, inflammation, and sometimes a loss of tissue function.

\section{At-Home and Surgical Treatment of Tissue Injury}

Treatment of these injuries starts with the PRICE (Protection, Rest, Ice, Compression, Elevation) method immediately following the injury: this involves remaining immobile and reducing pain and inflammation by applying ice and compression to the local area of the injury while elevating the injury above the heart (Hoffman, 2008). Once extent of injury is determined, mild injuries often go untreated to allow healing by natural processes where full recovery is likely to occur. Such mild injuries may include neuropraxia of nerve tissue caused by ischemia or intense compression, sprains and strains in connective tissues where tears are absent, mild burns of the outer skin, etc. Mild injuries can be treated with a continuation of the PRICE method until inflammation and pain cease.

If tissue injury is more severe and cannot be treated at home independently, surgery may be required to fix the injured tissue, and this often involves autografts or allografts from a donor site. For example, anterior cruciate ligament (ACL) injuries affect more than 200,000 people annually, and ACL reconstructive surgery is often the choice of treatment to return function as quickly as possible and improve quality of life (Kim and Smith, 2009). For ACL injuries that may require autografts, the surgeon will take tissue from other tendons, most often knee cap tendons or hamstring tendons, and secure the donor tissue in replacement of the damaged tissue (Ma, 2013). This, unfortunately, simply moves the wound site to an area that experiences less mechanical forces. Though allografts avoid the problem of an additional wound site in the same body, immunosuppression therapy is implemented in conjunction with allografts to minimize the chances of rejection of the donor tissue.

\section{Biomaterials Used in the Treatment of Tissue Injury}

With a research focus on tissue engineering, several different types of biomaterials have been synthesized and studied as an alternative to surgery to minimize invasive procedures while providing healing capabilities to a variety of injured tissues. 
For such purposes, biomaterials are constructed, ideally, to host cells and allow interaction with the living tissue environment, and this construct is called a scaffold; biomaterials that interact with living tissue are considered more ideal than inert materials because scaffolds have the potential to aid in more wound healing processes by hosting cells, drugs, and other healing factors (Keane and Badylak 2014). Scaffolds may include metals and ceramics, especially for orthopedic application, but the most popular type of biomaterial used in tissue engineering is the biodegradable polymer (Chen, Liang et al. 2013). These specific biomaterials are widely utilized due to their nature of degrading by the body's natural process and removing the need to perform invasive procedures to remove the material once its tissue engineering function is complete.

There are several examples of biodegradable polymers being studied for potential use in tissue engineering. In regards to neural tissue engineering, polymers such as Poly(3-hydroxybutyrate-co-3-hydroxyvalerate) (PHBV) utilized as a microsphere (Chen and Tong 2012) and chitosan substrates (Du, Tan et al. 2014) have shown effectiveness in neural progenitor cell (NPC) differentiation into neurons; to increase the efficiency of drug delivery, chitosan nanoparticles have been utilized for potential improved treatment of glaucoma (Katiyar, Pandit et al. 2014); hydrogels consisting of polyethylene glycol have shown promise as cell carriers for neural transplantation (Mahoney and Anseth 2006). In cardiac tissue, polysaccharide-based scaffolds have shown potential for improving cardiac function and repairing damaged tissue, especially following myocardial infarction in mice (Silva, Juenet et al. 2014). Even in bone tissue engineering, a focus still dominated by metals and ceramics, progress has been seen in bone repair and osteoblast differentiation using poly(fumaroyl bioxirane) maleate (PFM), a polymer that has mechanical strength range close to natural bone (You, Bi et al. 2012).

Biodegradable polymers that are naturally occurring are of particular interest in tissue engineering applications due to their inherent biocompatibility. Collagen is the most abundant protein found in the body, and its role of structural support in the ECM has made it a prime candidate for tissue engineering purposes. However, collagen is mechanically weak on its own, it varies in structure across species, and most major sources of collagen come from non-human animals. For these reasons, utilization of collagen may cause immunogenic responses to occur in patients (Chen, Liang et al. 2013). Some studies of naturally occurring long-chain polysaccharides have also had great success in tissue engineering applications. Chitosan, which is obtained from crustacean shells as one of the most abundant polymers on Earth, has had great success in several different tissue engineering applications, including differentiating neural stem cells as a hydrogel scaffold (Leipzig, Wylie et al. 2011) and improving cardiac function in conjunction with stem cells (Silva, Juenet et al. 2014). However, chitosan is not a native polymer of the human body. Hyaluronic acid is an abundant native polysaccharide of the human body, an advantage over non-native biopolymer choices, that is a highly conserved structure across species, which is a great advantage over collagen relating to immunogenic response (Price, Berry et al. 2007). For this reason, hyaluronic acid is one of the most studied biopolymers in recent years for application in tissue engineering. 


\section{Hyaluronic Acid}

\section{Role and Structure in the Body}

Hyaluronic acid (HA), also called hyaluronan, is a natural, carbohydrate polymer that has become an increasingly more important tool in the medical and bioengineering fields. Hyaluronic acid is a major component of the extracellular matrix (ECM), second only to collagen in terms of abundance. HA plays several roles in the body. Similar to collagen, it plays a role in structural integrity, connecting to proteoglycans of cells' plasma membranes and causing behavioral changes to occur. HA also plays an important role in lubrication of joints: hyaluronic acid is found in heavy concentrations in connective tissues and synovial fluid and aides in keeping the coefficient of friction low between contacting surfaces while ensuring the area is hydrated (Fraser, 1997). HA has even been proposed to have an important role in the development of the spinal cord, specifically cell migration and axonal growth (Meszar, Felszeghy et al. 2008). One of the most vital roles of HA is its involvement in the wound healing process. At low molecular weights, around $1500 \mathrm{Da}$, HA has been shown to aid in the delay of scar tissue formation resulting in complete healing of damaged areas: this is especially seen in infants, where concentration of low molecular weight HA is higher than those found in adults (Price, Berry et al. 2007) . At higher molecular weights, HA stimulates fibroblast proliferation which causes an increase in the production of collagen for constructing of ECM support in wounds (Price, Berry et al. 2007). When HA polymer chains reach lengths of 25 monomer units or below, HA is pro-angiogenic; therefore, it aides in the formation of new blood vessels to wound areas (Price, Berry et al. 2007). With its roles in structuring, lubrication, hydration, and wound healing, HA is a good candidate for utilization in biomedical applications.

HA, as a monomer unit, is composed of two carbohydrate molecules: Dglucuronic acid and $\mathrm{N}$-acetylglucosamine, which interchange along the length of HA polymer chains. The molecular weight of HA chains varies widely in the body, from hundreds to multiple millions of Daltons. Production of HA occurs in the plasma membrane of fibroblasts, and the deposition and reuptake of HA from and into fibroblasts is controlled by the CD44 receptor on the surface of the cell (Price, 2007; Fraser, 1997). For the average 70 kilogram person, there is roughly 15 grams of HA in their body at any given time. However, HA is highly recycled, and it has a turnover rate of 3 days (Stern, 2004). HA is a glycosaminoglycan, and it is often seen as a sodium salt in physiological conditions due to its negative charge (Vasi, 2014). HA is highly hydrophilic, forming a gel-like consistency when interacting with water, which aides in its roles of hydration and lubrication in connective tissues. Though HA is not as prevalent as collagen in the ECM, HA has a very distinct property that gives it an advantage when it comes to prevalence: the structure of HA is highly conserved across different species and even phyla, thus extracted HA from sources other than humans have identical structure (Price, 2007).

Because HA is a natural polymer produced in the body with a highly conserved structure, it is biocompatible. Through enzymatic action and CD 44 receptor interaction 
of fibroblasts, HA is also biodegradable and bioresorbable. With its functional groups, such as carboxylic acid and hydroxyl groups, HA is able to be conjugated with other molecules in order to obtain various mechanical, rheological, and physical properties. Because of these characteristics, including the various roles and most importantly wound healing, HA is a reasonable choice for utilization in scaffolding for the purpose of tissue engineering.

\section{Commercial Uses of Hyaluronic Acid as a Scaffold}

HA is largely obtained from rooster combs due to the high concentration of HA found there, up to $7.5 \mathrm{mg} / \mathrm{mL}$, but it also can be obtained from bacteria-streptococci (Collins, 2013). Products such as Hyalogran $\bigcirc$ are used for pain relief from underlubricated joints and contain high molecular weights of HA to prolong the treatment. HA has also been used in wound dressing applications, often manufactured as a sheet to be used simultaneously with bandages (Price, Berry et al. 2007). The wound dressing product Hyalofill (C) utilizes HA's gelation ability in this way, which maintains a wet environment in wounds under bandaging to ensure proper healing. In addition, hyaluronic acid has shown potential as an effective dermal filler and a potential treatment for osteoarthritis (Fakhari and Berkland 2013).

\section{Hyaluronic Acid Structure Modification for Scaffold Use}

The use of HA as a scaffold is done mostly in conjunction with another functional molecule because HA is mechanically weak on its own (Fakhari and Berkland 2013). HA is highly hydrophilic, and thus dissolves easily in water. In addition, natural enzymes, such as hyaluronidase, and the re-uptake of HA by fibroblasts increases the degradation rate of HA in the body (Price, Berry et al. 2007). Though this works well with viscosupplementation treatment, other applications that require scaffolds to degrade over periods of days, weeks, and months must have HA that is modified. One modification that has been utilized in several studies is the methacrylation of HA, allowing for the cross-linking of HA polymer chains and the lowering of degradation rates of HA (Bencherif, 2008; Schante, 2011; Bae, 2011; Vasi, 2014; Nguyen, 2014). This is achieved primarily through conversion of HA in tetrabutylammonium (TBA) form and conjugation of a methacrylate group to HA in an organic solvent (Oh, Park et al. 2010). Once HA has been modified with a methacrylate group, it is able to be cross-linked either chemically or through use of UV, called photopolymerization. For photopolymerization, cross-linking is done by using a photoinitiator, usually Irgacure 2959 (Bae, 2011; Schante, 2011; Nguyen, 2014) and the result is a network of polymer chains called a hydrogel. Hydrogel scaffolds are hydrophilic and can swell up to 1000 times their dry weight in an aqueous environment, yet the hydrophobic crosslinks protect the structure from hydrolysis and enzymatic degradation (Seliktar 2012). This type of scaffold is useful in the delivery and carrying of cells because it allows the aqueous environment to enter while providing structural integrity, thus mimicking the 3D environment of the ECM (Tibbitt and Anseth 2009). In addition, encapsulation of cells within hydrogels is possible because the method 
of synthesizing the scaffold, whether it is covalent or physical methods, can be done under mild conditions to ensure maximizing cell survival (Guvendiren and Burdick 2013). Cross-linking HA makes for a stronger hydrogel that can last longer, and modifications of the cross-linker can help control these properties.

\section{HA-Based Hydrogels for Tissue Engineering Purposes}

In regards to tissue engineering, HA-based hydrogels have shown potential in various tissue engineering applications, especially in conjunction with various omni- and pluripotent stem cells. Studies done in 2013 study by Liming Bian and Iris Kim showed that HA conjugated with methacrylic anhydride formed a hydrogel that had improved compressive strength as crosslinking density increased and successfully induced chondrogenesis of mesenchymal stem cells (Bian, 2013; Kim, 2013). For applications involving cardiac tissue, HA hydrogels crosslinked with N-hydroxysuccinimide (NHS) mixed with blood have shown success in proliferation of cardiosphere derived cells (CDCs) and improving cardiac function after injection in rats (Chang, 2012).

Conjugations of HA with tyramine have even shown roles in organization of bone matrix, controlling cellular condensation during chondrogenesis, and differentiation of MSCs (Toh, Lim et al. 2012). HA hydrogels have shown potential to successfully encapsulate and maintain non-differentiation of embryonic stem cells (Gerecht, Burdick et al. 2007). Furthermore, methacrylated HA hydrogels have also been successful in the proliferation of neural progenitor cells (NPCs) and their differentiation into neuron- and astrocyte-like cells (Seidlits, 2010). For HA-AEMA hydrogels, studies have found success of these scaffolds to aid in osteogenesis (Bae, 2011; Bae, 2014). Though studies have a variety of methacrylate groups conjugated to the HA backbone to form the hydrogel for different tissues applications, being able to tune the mechanical properties of one specific macromer to span a broad range of strengths may also be influenced by molecular weight of the backbone (in this case, HA).

Mechanical properties of HA-based hydrogels have also been studied under various conditions. A 2008 study by Siri Bencherif showed that HA hydrogels crosslinked with glycidyl methacrylate had increasing compression moduli as the crosslinking density was increased (Bencherif, 2008). Many studies involving HA-based hydrogels utilize only one molecular weight of HA when analyzing the mechanical properties of hydrogels, and the only mechanical property characterized for these studies is the compressive modulus. To gain a better understanding of the mechanical properties of these 3D hydrogels, it is important to evaluate their response to other mechanical forces that may be present, such as shear forces. In the $3 \mathrm{D}$ in vivo environment, hydrogels may experience stresses ranging from the very soft brain environment $(\sim 0.1$ $\mathrm{kPa})$ to stiff bone ( $\sim 80 \mathrm{kPa})$ (Guvendiren and Burdick 2013). Native tissue have elastic moduli ranging from $0.5 \mathrm{kPa}$ and $10^{10} \mathrm{~Pa}$, respectively (Li, Katsanevakis et al. 2012), and these tissue environments experience both shear and normal forces. Therefore, this work characterizes HA-AEMA hydrogel mechanical properties both in compression and shear, and hydrogels differ both in molecular weight and crosslinking density to analyze the effects of multiple tunable factors on the strength of the hydrogel system. Furthermore, 
this work also checks the viability of these HA-AEMA hydrogels for tissue engineering application by exposing and encapsulating DPSCs in these systems. Studies have shown that DPSCs, which can be obtained easier than other NPCs due to their location outside the CNS in adults (Gronthos, S. et. al. 2000; Huang, G.T. et al. 2009) and have the potential to differentiate in to several different types of cells, including osteoblasts, odontoblasts, neuronal cells, epithelial cells and others given the right conditions (Kawashima 2012; Arthur A. et. al, 2008), thus successfully encapsulating DPSCs with HA-AEMA hydrogels would show great potential in utilizing this particular system to many different applications of tissue engineering across the human body. 


\section{CHAPTER 3. MATERIALS AND METHODS}

\section{Synthesize and Characterize HA-AEMA Macromer}

\section{Synthesis Process of HA-AEMA Macromer}

Ion Exchange. Sodium salt configuration of hyaluronic acid (HA), specifically molecular weights of $18 \mathrm{kDa}$ and $270 \mathrm{kDa}$, underwent ion exchange and was converted to its tetrabutylammonium (TBA) form. Specifically, 30 grams of Dowex-8X-50 ion exchange resin was washed three times with $500 \mathrm{~mL}$ of deionized (DI) water and dried by vacuum filtration. After washing, the resin was mixed with 50 milliliters of $1.5 \mathrm{M}$ TBA and 25 milliliters of DI water to convert the ion exchange resin into TBA form and was left to stir overnight. After overnight mixing, a solution of sodium salt-type hyaluronic acid in DI water, with a concentration of $10 \mathrm{mg} / \mathrm{mL}$, was added to the converted resin and mixed for 24 hours. This allows for the exchange of sodium salt on HA with TBA to form HA-TBA. After mixing, the mixture was vacuum-filtrated, and the ion exchange resin was washed three times with DI water to ensure maximum collection of remaining supernatant. After vacuum filtration, the ion exchange resin was mixed with fresh TBA and DI water for another cycle of conversion, and the collected supernatant was lyophilized for 72 hours to obtain HA-TBA in solid form. The ion exchange process is necessary in order for HA to dissolve in dimethyl sulfoxide (DMSO), which is not possible in the sodium salt state. ${ }^{1} \mathrm{H}$ NMR was utilized to confirm synthesis of HA-TBA.

AEMA Conjugation. HA-TBA macromer was collected following the lyophilization step of the ion exchange process and dissolved in DMSO. The amount of DMSO used to completely dissolve the HA-TBA macromer was kept to a minimum for the ease of later steps in the synthesis process, and this amount varies dependant on the molecular weight of HA. The dissolution process was done in the presence of nitrogen to maintain air flow and minimize water intrusion from the ambient air. After complete dissolution of HA-TBA, three components were added to the solution: AEMA, benzotriazol-1-yl-oxytripyrrolidinophosphonium hexafluorophosphate (PyBOP), and diisopropylethylamine (DIPEA). Prior to the addition of these components, AEMA and PyBOP were brought to room temperature, dissolved completely in DMSO at a concentration of $100 \mathrm{mg} / \mathrm{mL}$, and added to the solution. DIPEA was added last. To achieve a degree of substitution of $25 \%$, the ratio of HA to component, per molar concentration, was 1:4 for all three components added. After adding the components to the DMSO solution, the air flow of nitrogen gas was removed and the mixture was sealed. The mixture is shielded from light and allowed to stir for 7 days to allow for maximum conjugation.

Precipitation. One day prior to the end of the stirring cycle of the conjugation step, a volume of acetone, equal to 10 times the volume of the DMSO solution, was chilled in a freezer. After chilling overnight, the acetone was set in an ice water bath, and the DMSO solution was added drop-wise into the acetone. Once all of the solution had 
been added, the precipitate was allowed to settle to the bottom of the precipitation beaker for 15 minutes. Excess acetone was removed from the beaker after settling, the precipitate was resuspended, and then the precipitate mixture was divided equally among a number of centrifuge tubes. The divided mixture was centrifuged for 5 minutes, and the acetone liquid phase of the mixture was carefully discarded. Fresh acetone was added to each of the centrifuge tubes, and the precipitate mixture was resuspended and centrifuged again. This cycle was done three times to remove any excess DMSO still present. After the acetone washings, acetone was removed from all of the tubes, and the precipitate was combined.

During one attempt at synthesis, an additional step was added after the above precipitation steps as an experiment: the precipitate was vacuum dried for 24 hours prior to beginning dialysis. Visual comparisons were made between the final product of this synthesis procedure and other products of syntheses without including this step.

Dialysis and Final Product. The precipitate was completely dissolved in DI water. The solution was then transferred to a dialysis tub with a molecular weight cut-off of 6000-8000 Da. Once the dialysis membrane was sealed, it was suspended in a $3 \mathrm{~L}$ container of $1.5 \mathrm{M} \mathrm{NaCl}$ in DI water, and the suspension solution was stirred for 2 hours while hidden from light. After 2 hours, the salt solution was replaced, the membrane was resuspended, and the suspension solution was stirred for an additional 2 hours. This process was repeated once more, and at the six hour mark of dialysis, the salt solution was replaced with only DI water. The suspension solution was replaced as often as possible with at least 2 hours time passing between each replacement. After dialysis was performed for 24 hours, the contents of the membrane were transferred to a freeze-drying container and lyophilized for 72 hours. The solid product of the final lyophilization was HA-AEMA macromer.

\section{Characterization of HA-AEMA Macromer}

Characterization of Macromer Structure. After synthesis was completed, HAAEMA macromer structure was characterized by ${ }^{1} \mathrm{H}$ NMR using a Bruker $400 \mathrm{MHz}$ NMR spectrometer. Macromer was dissolved in deuterium oxide $\left(\mathrm{D}_{2} \mathrm{O}\right)$ at a concentration of $10 \mathrm{mg} / \mathrm{mL}$. After dissolution, analysis was performed via proton experiment with the number of iterations set to 128 . In addition to identifying characteristic peaks, actual degree of substitution was calculated by peak integration comparison.

Macromer Solubility. HA-AEMA macromer was dissolved in DI water to test solubility at concentrations of $0.1 \mathrm{wt} \%, 0.5 \mathrm{wt} \%, 1 \mathrm{wt} \%, 2 \mathrm{wt} \% 3 \mathrm{wt} \%$, and $5 \mathrm{wt} \%$. Several macromer types were tested, differing in HA molecular weights $(6.4 \mathrm{kDa}, 16 \mathrm{kDa}, 18 \mathrm{kDa}$, $37 \mathrm{kDa}, 66 \mathrm{kDa}, 270 \mathrm{kDa})$ and degree of AEMA substitution $(12.5 \%, 25 \%$, and $37.5 \%)$. The macromers were allowed to stir for, at most, 4 hours, and any macromer that did not fully dissolve within this time frame were considered 'insoluble'. 
Viscosity of Macromer in Solution. Synthesized HA-AEMA macromer was dissolved in a solution of DI water and Irgacure 2959 (0.05\%). Once dissolved, the viscosity of the resulting macromer solution was tested on an AR-G2 rheometer from TA instruments by performing a oscillating shear rate ramp at a constant temperature of $37^{\circ} \mathrm{C}$ on $70 \mu \mathrm{L}$ of the macromer solution. The software associated with the rheometer was set up to take measurements of the viscosity periodically as the shear rate increased, and a resulting graph was created upon termination of the shear ramp. The viscosity of the macromer solution was selected as an average of the viscosity values on the resulting graph which were independent of the shear rate applied to the solution. The experiment was repeated 3 times for every batch of macromer solution created. Macromer concentration was $3 \mathrm{wt} \%$, and the viscosities of macromer solutions HA-AEMA-16k-25, HA-AEMA-66k-25, and HA-AEMA-270k-25 were compared.

\section{Synthesize and Characterize HA-AEMA Hydrogels}

\section{Formation of HA-Based Hydrogels}

Hydrogel Formation via UV Polymerization. HA-AEMA macromers that were successful in completely dissolving in DI water at various concentrations in less than 4 hours were dissolved again in $\mathrm{H}_{2} \mathrm{O}$ and were exposed to UV light at an intensity of 360$390 \mathrm{~mW} / \mathrm{cm}^{2}$ for 10 minutes at each of the concentrations previously tested. Irgacure 2959, a biocompatible photoinitiator, was added to the $\mathrm{H}_{2} \mathrm{O}$ solution at a concentration of $0.05 \mathrm{wt} \%$ prior to dissolving the macromers to initiate cross-linking. $50 \mathrm{uL}$ of macromer solution was placed into a Teflon well and exposed to UV, and, if a hydrogel was successfully formed, it was carefully removed from the well and air-dried on a non-stick surface for 24 hours. Macromer solutions that did not form a hydrogel after the first exposure were exposed at most twice more for the same duration before considering it a 'failure'.

Analyze Swelling Ratio of HA-based Hydrogels. Hydrogels formed from the successful macromers were weighed and submerged in PBS following the air-drying process and kept hidden from light. Visual checks were performed on the hydrogels to see if they were still present in the PBS from the time of initial submersion. Swelling ratios for hydrogels were calculated according to Equation 3-1:

$$
\text { Swelling Ratio }=\left(\mathrm{W}_{\text {wet }}-\mathrm{W}_{\mathrm{dry}}\right) / \mathrm{W}_{\mathrm{dry}} * 100 \%
$$

\section{Investigation of Mechanical and Rheological Properties of HA-Based Hydrogels}

Storage and Loss Moduli Measurement. Hydrogels were formed following the steps described above. Following immersion in PBS, the both the storage modulus and loss modulus of hydrogels were tested using an AR-G2 TA Instruments Rheometer. Specifically, hydrogels were subjected to an oscillating strain ramp, from 0.1 to 10 
percent strain on a logarithmic scale, while maintaining normal force to $0.01-0.03 \mathrm{~N}$, the temperature at $37^{\circ} \mathrm{C}$, and a constant $1 \mathrm{~Hz}$ frequency of oscillation. The software for the rheometer was set up to take 20 sample points per decade of strain, and a resulting graph of the storage modulus over the range of strain was created at the termination of the procedure. Storage modulus was measured as the average value of data points along the linear viscoelastic region (LVR), or the section of the graph in which storage modulus was independent of the strain. Comparisons of storage moduli were made between hydrogels that differed in several different categories, described below.

HA Molecular Weight. The storage moduli of hydrogels that differed in the molecular weight of the HA component of the macromer were compared using Student T-test to check for significant differences. Molecular weights that were included were 16 $\mathrm{kDa}, 18 \mathrm{kDa}, 66 \mathrm{kDa}$, and $270 \mathrm{kDa}$.

AEMA Degree of Substitution. The storage moduli of hydrogels that differed in the degree of substitution of the AEMA component of the macromer were compared using Student T-test to check for significant differences. Degree of substitutions that were included were $12.5 \%$ and $25 \%$.

Macromer Concentration in Photoinitiator Solution. The storage moduli of hydrogels that differed in the macromer concentration of the photoinitiator solution were compared using Student T-test to check for significant differences. Concentrations that were included were $3 \mathrm{wt} \%$ and $5 \mathrm{wt} \%$

Following measurement of the storage modulus using the strain ramp procedure, measurement of both the storage and loss moduli was done during a frequency sweep using the rheometer. While maintaining a temperature of $37^{\circ} \mathrm{C}$ and a sampling rate of 20 points per decade, the frequency of oscillation varied from $0.1 \mathrm{~Hz}$ to $100 \mathrm{~Hz}$ with a constant strain of $1 \%$. Once storage modulus measurements indicated a drastic drop in elasticity of the hydrogel, the sweep was stopped, and the frequency at which the storage modulus began to drop off was considered the critical frequency.

Compressive Strength Measurement. Following hydrogel swelling, the compression profile of HA-AEMA-18k-25 hydrogels were evaluated following swelling in PBS at time 0 (immediately following UV polymerization), 1 day, 3 days, 7 days, 15 days, 21 days, and 28 days using a TA Instruments Dynamic Mechanical Analyzer (DMA) Q800. The DMA was calibrated before each set of experiments, and the dimensions of the hydrogels were measured and recorded in the accompanying DMA software prior to testing. Starting at a normal force of $0.001 \mathrm{~N}$, the hydrogel was heated to $37^{\circ} \mathrm{C}$ and put under a force ramp of $0.25 \mathrm{~N}$ per minute until the machine reached $3 \mathrm{~N}$ or the experiment automatically stopped due to rupture of the hydrogel. After the force ramp was complete, a graph of the resulting stress/strain curve was produced. To determine the Young's modulus of the compressed hydrogel, the slope of the stress/strain curve was analyzed at a point where the profile was linear and most consistent. Graphs were produced comparing the profiles and moduli of each swelling time point. In addition, 
HA-AEMA-270k-25 hydrogels were compressed under the same conditions immediately following UV polymerization to compare results with HA-AEMA-18k-25 hydrogels.

\section{Study Cytotoxicity and Encapsulation Capabilities of HA-AEMA Macromers and Hydrogels}

\section{Cytotoxicity of HA-AEMA Macromer to DPSCs}

Cytotoxicity of HA-AEMA-18k-25 and HA-AEMA-270k-25 macromers to human DPSCs was tested using MTT mitochondrial based assays purchased from ATCC. Human DPSCs (obtained from Dr. George Huang's lab, UTHSC) at 3rd passage were seeded at 15000 cells per cm 2 in 96 well plates and incubated for 24 hours. Macromer solution was added to each of the test wells in concentrations of $0.5 \%, 1 \%$, and $3 \%$. Growth medium, which consisted of $\alpha$-MEM, 10\% FBS, $100 \mathrm{U} / \mathrm{mL}$ streptomycin, 100 $\mathrm{U} / \mathrm{mL}$ penicillin, and $25 \mathrm{ng} / \mathrm{mL}$ amphotericin $\mathrm{B}$, was added to the control wells. Exposure of DPSCs to macromer was limited to 30 minutes to simulate short-term exposure found in the encapsulation study protocol, and MTT reagent was added to all wells following this duration. The plate was then incubated for a further 4 hours, at which point detergent reagent $(10 \% \mathrm{SDS}$ in $0.01 \% \mathrm{HCl} /$ water solution) was added to all wells. After incubating at room temperature in the dark for another 2 to 4 hours, absorbance of each well was measured on a microplate reader at a wavelength of $550 \mathrm{~nm}$. Because absorbance readings of MTT assay was strongly correlated with cell viability (linear positive relationship), ratios of absorbance of testing wells to absorbance of control wells can give percentages of cell viability.

\section{DPSC Survival in HA-Based Hydrogels}

DPSCs were grown until confluent in a $25 \mathrm{~cm}^{2}$ culture flask and trypsinized for cell counting. Cell concentration was diluted and loaded into HA-AEMA macromer, both $18 \mathrm{k}-25$ and $270 \mathrm{k}-25$ hydrogel solutions, at $2.5 \times 10^{4}$ cells per hydrogel volume $(50 \mathrm{uL})$. Immediately following UV polymerization for 10 minutes, hydrogels were suspended in growth medium and incubated for 24 hours. After incubation, growth medium was removed and replaced with calcein AM for 30 additional minutes for staining live cells. After incubation, hydrogels were imaged at $4 \mathrm{X}$ magnification.

\section{Statistical Analysis}

Data were reported as mean \pm standard deviation (S.D.) from at least three separate experiments for the viscosity, storage/loss modulus, compressive strength, and cytotoxicity studies. Two-tailed Student's t-test was used to analyze the differences between treatment groups. A statistically significant difference was reported if $p=0.05$ or less. 


\section{CHAPTER 4. RESULTS}

\section{Synthesis and Characterization of HA-AEMA Macromer}

\section{Macromer Synthesis}

Ion Exchange. A visual inspection of the sodium salt-type HA, prior to the beginning of synthesis, revealed that the lower molecular weight $18 \mathrm{kDa}$ was courser, while the $270 \mathrm{kDa} H A$ was a finer white powder. Dissolution of HA in $100 \mathrm{~mL}$ of DI water took significantly longer for the $270 \mathrm{kDa}$ molecular weight over $18 \mathrm{kDa}$, specifically 15 minutes and 3 minutes, respectively. Since the same mass of HA powder was measured for both the $18 \mathrm{kDa}$ and $270 \mathrm{kDa}$ molecular weight, the molar concentration of HA in water is higher for the $18 \mathrm{kDa}$; however, ion exchange resin is utilized in excess to ensure maximum ion exchange for both molecular weights.

Following the 24 hour mixing period for ion exchange to take place, the supernatant for the $270 \mathrm{kDa}$ appeared more viscous than the $18 \mathrm{kDa}$ supernatant. Both supernatants were collected following vaccum filtration and lyophilized. Samples from each batch were taken following lyophilization, dissolved in DMSO, and analyzed by ${ }^{1} \mathrm{NMR}$. Confirmation of successful ion exchange, resulting in the formation of HA-TBA, can be seen in Figure 4-1. Specifically, the methyl and methylene groups associated with TBA are seen at $0.9 \mathrm{ppm}$ and $1.3 \mathrm{ppm}$, respectively, and the ester bond located on the glucosamine unit of HA is shown at 1.5-1.6 ppm.

AEMA Conjugation. A visual inspection of the HA-TBA macromer following lyophilization revealed that the lower molecular weight $18 \mathrm{kDa} H A-T B A$ appeared to be thinner in substance and stringier. For the higher molecular weight $270 \mathrm{kDa}$, it looked much thicker and significantly less porous.

Dissolution of both macromers in DMSO were successful, but it takes about 40 $\mathrm{mL}$ of DMSO to completely dissolve the $270 \mathrm{kDa}$ macromer in comparison with just 15 $\mathrm{mL}$ to completely dissolve the $18 \mathrm{kDa}$ macromer. In addition, the lower molecular weight HA-TBA also took a significantly shorter time period to completely dissolve in DMSO over the higher molecular weight HA-TBA. However, the added components, AEMA, PyBOP, and DIPEA, are dependent only on the initial mass of HA used in synthesis, the molecular weight of the HA monomer, and the desired degree of substitution of the AEMA component. Because we used 1 gram for each molecular weight and our desired degree of substitution for each was $25 \%$, the same amount of each component was added during the conjugation step.

Precipitation. For both molecular weights of macromer added dropwise to the chilled acetone, the precipitate that formed upon addition looked similar to each other: a translucent gel suspension. However, once the excess acetone was removed and the precipitate was centrifuged, the precipitate was a white gelatinous solid at both molecular weights. 


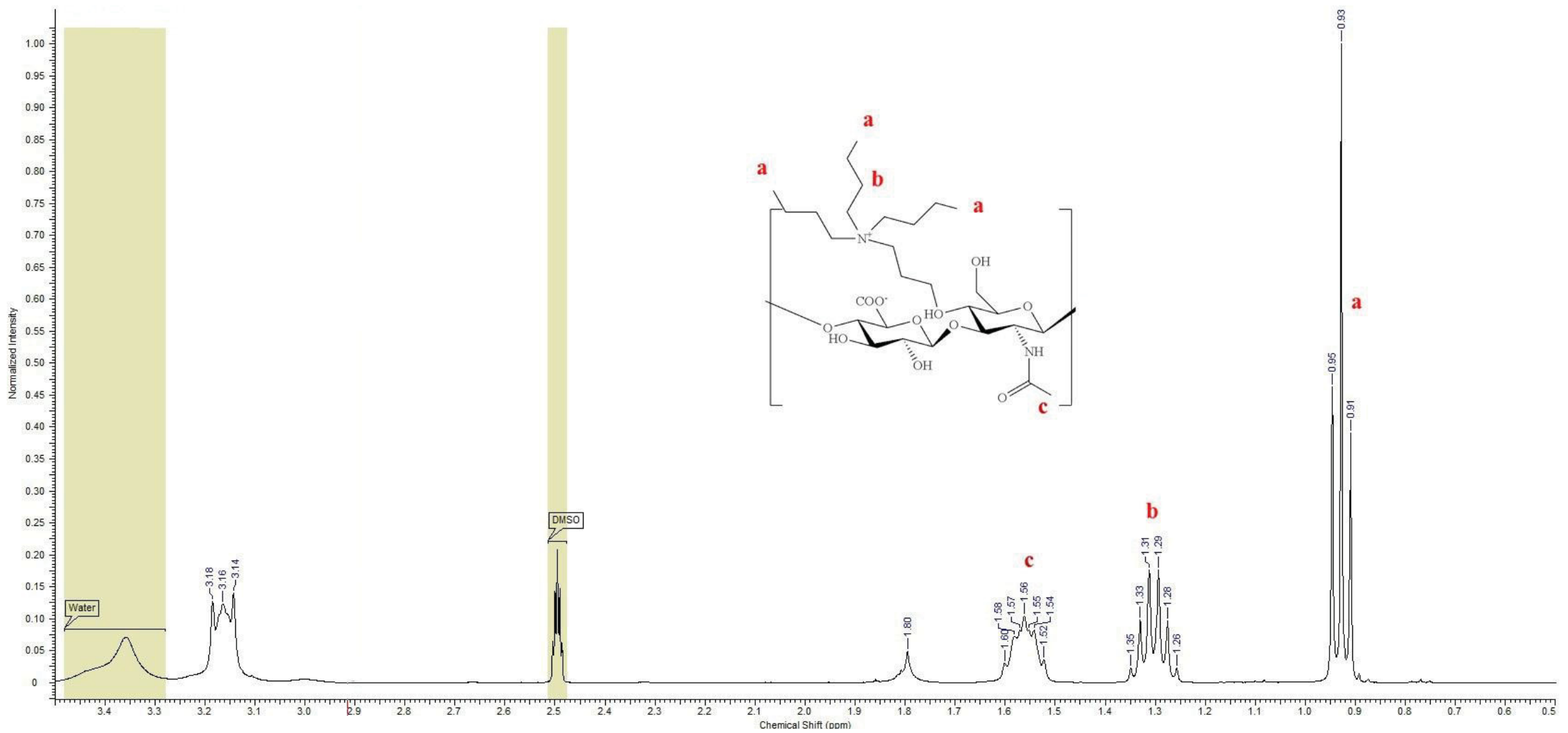

Figure 4-1. $\quad{ }^{1}$ NMR of HA-TBA following ion exchange process of synthesis.

The characteristic peaks for TBA are located around $0.9 \mathrm{ppm}$ (methyl group, label ' $a$ ') and $1.3 \mathrm{ppm}$ (methylene group, label ' $b$ ') with the characteristic ester bond for HA shown around 1.5-1.6 ppm (ester bond, labeled 'c'). The other groups of peaks are characteristic for HA, both of which match for each molecular weight. 
In one synthesis attempt, the precipitate was dried in a vacuum for 24 hours following centrifuging in an attempt to completely remove the acetone prior to DI water dissolution and dialysis. However, this resulted in almost complete loss of the precipitate for that $270 \mathrm{kDa}$ molecular weight batch, and no end product could be obtained. There was some loss of the $18 \mathrm{kDa}$ molecular weight batch that was vacuum dried after centrifuging, but a final product at the end of synthesis was created. The vacuum dried batch of $18 \mathrm{kDa}$, seen on the left in Figure 4-2, did not form a hydrogel when the prepared macromer solution was exposed to UV light. However, the batch of $18 \mathrm{kDa}$ that was not vacuum dried, seen on the right, did form a hydrogel.

Dialysis and Final Product. Dialysis of the precipitate was performed for only 24 hours. Earlier attempts at synthesis had dialysis being performed for 3 to 5 days, yet the end result would fail to make hydrogels. Samples were taken after lyophilization of the dialysis solution and ${ }^{1} \mathrm{H}$ NMR shows successful synthesis of HA-AEMA macromer for both molecular weights, seen in Figure 4-3. The final product is white with a cottonlike consistency for both batches, seen on the right side of Figure 4-2. The total mass of the final product for the $18 \mathrm{kDa}$ batch and the $270 \mathrm{kDa}$ batch were $237 \mathrm{mg}$ and $250 \mathrm{mg}$, respectively. The biggest contributor to the loss of mass throughout synthesis is the precipitation step, where the amount of precipitate obtained following the dropwise addition into acetone is much less than the amount of HA-TBA obtained following ion exchange.

\section{HA-AEMA Macromer Characterization}

Characterization of Macromer Structure. Graph of the NMR data for HAAEMA-18k-25 can be seen in Figure 4-3. In order to determine the degree of substitution of AEMA to the HA polymer, the area under the curve (AUC) for the characteristic peaks of the methyl groups for HA (located on the N-acetyl glucosamine unit) and AEMA were compared, resulting in a ratio of 6:1, respectively, and a degree of substitution between $16-18 \%$. Furthermore, the split characteristic peaks of the carboncarbon double bond on AEMA were present at chemical shifts of both 5.7 and $6.1 \mathrm{ppm}$, further indicating successful conjugation. In regards to HA-AEMA-270k-25, degree of substitution calculations of AEMA also resulted in 16-18\% substitution.

Macromer Solubility. In addition to performing solubility tests on the two synthesized macromers, The results of the solubility experiment on several different HAAEMA macromers can be seen in Table 4-1. Of the seven macromers tested for solubility in PBS, only HA-AEMA-37k-37.5 was insoluble in all concentrations, even after 24 hours of mixing. Since there were macromers of HA molecular weights exceeding that of the insoluble macromer that were soluble themselves, the limiting factor of solubility was the increased degree of substitution of the AEMA component. All other macromer concentrations were completely dissolved well under the 4 hours cutoff mark. The longest duration before complete dissolution was recorded for HA-AEMA270k-25, which took around 15 minutes at 5\% concentration. HA-AEMA-66k-25 was the next longest with 10 minutes at the $5 \%$ concentration, but all other macromers completely 


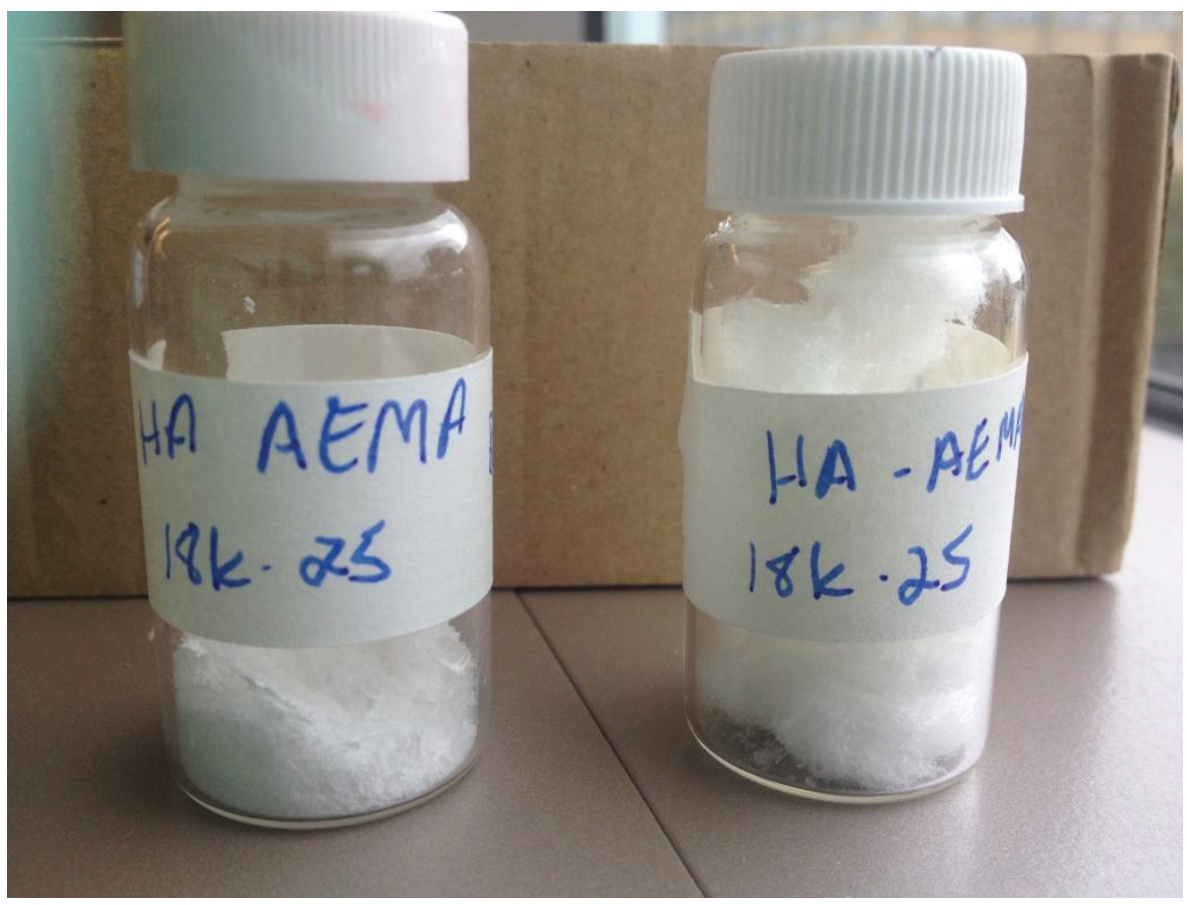

Figure 4-2. Vacuum drying precipitate affects end product of synthesis. Left: Vacuum dried during precipitation. Right: Normal procedure. The end product becomes flaky and fails to produce a hydrogel for the vacuum dried procedure, whereas the normal procedure is very fluffy and produces a hydrogel. 


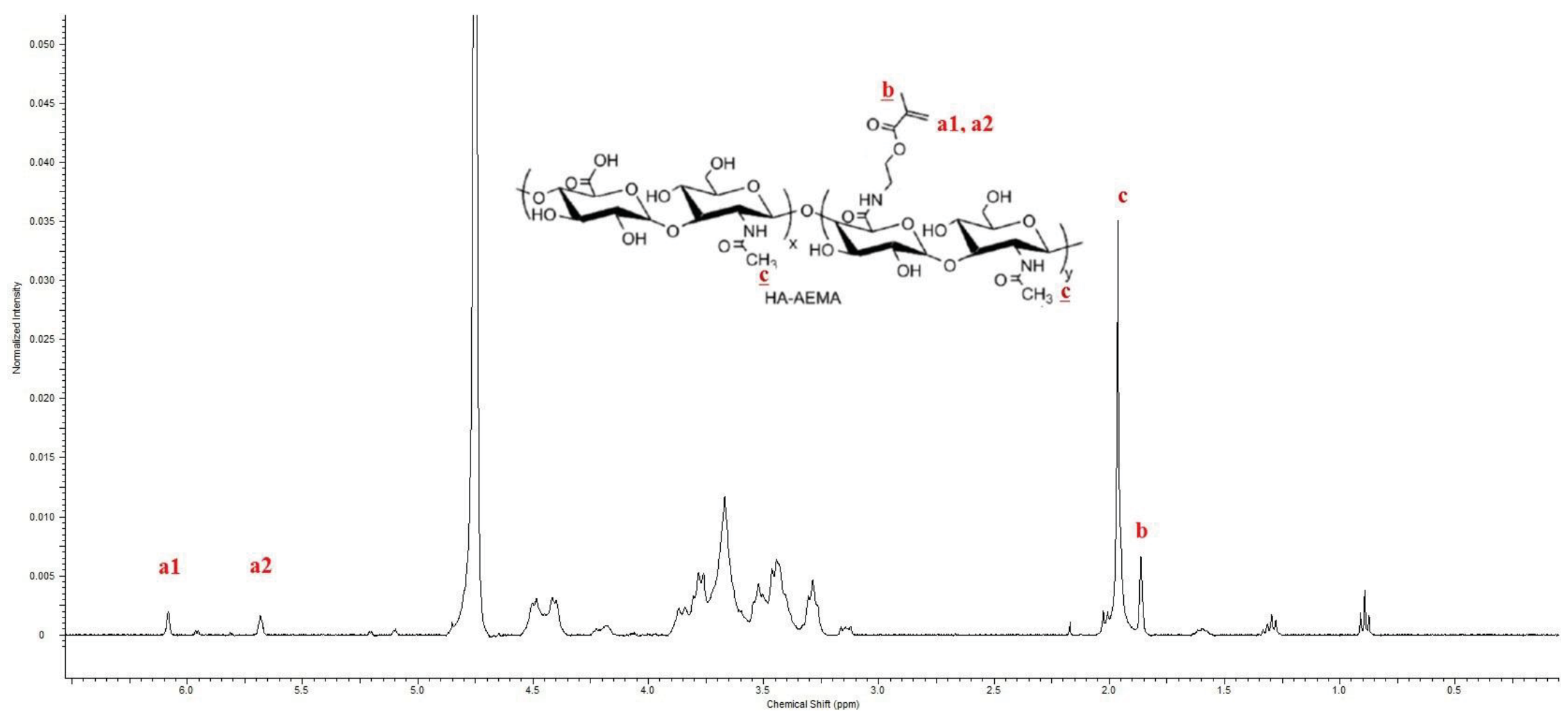

Figure 4-3. NMR graph of HA-AEMA-18k-25 for structure characterization.

NMR data confirms successful conjugation of AEMA to the HA polymer backbone. To calculate the degree of substitution of AEMA, the peak intensity of the methyl groups for AEMA and HA (labeled b and c, respectively) were compared and were found to be 1:6, resulting in an actual degree of substitution around 16-18\%. Peaks clustered around $3-4$ ppm are characteristic for HA 
Table 4-1. Solubility test of HA-AEMA macromers in PBS.

\begin{tabular}{|c|c|c|c|c|c|c|}
\hline \multirow{2}{*}{ Macromer } & \multicolumn{6}{|c|}{ Concentration of Macromer in PBS (wt\%) } \\
\hline & 0.1 & 0.5 & 1 & 2 & 3 & 5 \\
\hline $\begin{array}{l}\text { HA-AEMA-6.4k- } \\
25\end{array}$ & Soluble & Soluble & Soluble & Soluble & Soluble & Soluble \\
\hline $\begin{array}{l}\text { HA-AEMA-16k- } \\
25\end{array}$ & Soluble & Soluble & Soluble & Soluble & Soluble & Soluble \\
\hline $\begin{array}{l}\text { HA-AEMA-18k- } \\
25\end{array}$ & Soluble & Soluble & Soluble & Soluble & Soluble & Soluble \\
\hline $\begin{array}{l}\text { HA-AEMA-37k- } \\
37.5\end{array}$ & Insoluble & Insoluble & Insoluble & Insoluble & Insoluble & Insoluble \\
\hline $\begin{array}{c}\text { HA-AEMA-66k- } \\
12.5\end{array}$ & Soluble & Soluble & Soluble & Soluble & Soluble & Soluble \\
\hline $\begin{array}{l}\text { HA-AEMA-66k- } \\
25\end{array}$ & Soluble & Soluble & Soluble & Soluble & Soluble & Soluble \\
\hline $\begin{array}{l}\text { HA-AEMA-270k- } \\
25\end{array}$ & Soluble & Soluble & Soluble & Soluble & Soluble & Soluble \\
\hline
\end{tabular}


dissolved in under 1 minute across all concentrations. Based on these results, the degree of substitution of the AEMA component of the macromer is a much more important factor in determining the macromer's solubility.

\section{Viscosity}

For all three macromers used in the viscosity studies, there is a settling of the viscosity around a strain rate of $10 \mathrm{~Hz}$ and beyond, exhibiting characteristics of shear thinning as the oscillating strain rate increases. For HA-AEMA-16k-25, HA-AEMA-66k25, and HA-AEMA-270k-25 the viscosity was measured as $0.0131 \mathrm{~Pa}-\mathrm{s}, 0.0383 \mathrm{~Pa}-\mathrm{s}$, and $0.0560 \mathrm{~Pa}-\mathrm{s}$ respectively, seen in Figure 4-4. For each increasing molecular weight, there is a significant increase in the viscosity: the p-values calculated from comparisons of $16 \mathrm{k}-25: 66 \mathrm{k}-25$ and $66 \mathrm{k}-25: 270 \mathrm{k}-25$ were $\mathrm{p}<0.01$ and $\mathrm{p}<0.05$, respectively. Therefore, there is a direct relationship between the molecular weight of the HA component of the macromer and the viscosity of the macromer solution. Higher molecular weight of HA in macromer results in higher viscosity, which may be helpful in cases where a macromer solution needs to hold a temporary shape during the UV polymerization process to form a hydrogel. If the viscosity of the macromer solution is high enough, UV polymerization of the macromer may be able to be performed directly on damaged nerve tissue rather than have implantation of a previously formed hydrogel on the site.

\section{Synthesis and Characterization of HA-AEMA Hydrogels}

\section{Hydrogel Formation via UV Polymerization}

The results of the hydrogel formation tests can be seen in Table 4-2. Of all the macromers that were able to completely dissolve in PBS, only HA-AEMA-6.4k-25 did not form hydrogel at any concentration, even after 30 minutes of UV exposure. Because hydrogels were able to form from macromers that contained a lower degree of substitution (HA-AEMA-66k-12.5), the limiting factor for HA-AEMA-6.4k-25 was the molecular weight of HA, therefore there is a lower limit at which hydrogels cannot be created. Perhaps HA-AEMA-6.4k-25 may be able to form hydrogels at higher degrees of substitution for the AEMA component, but solubility would have to be tested. In addition to HA-AEMA-6.4k-25 not forming hydrogels, all concentrations below $2 \%$ also did not form a hydrogel for any of the macromers. From this information, it can be concluded that the critical concentration for which a hydrogel can be formed lies between $1 \%$ and $2 \%$. At $2 \%$ and greater concentrations, hydrogels formed for all macromers. 


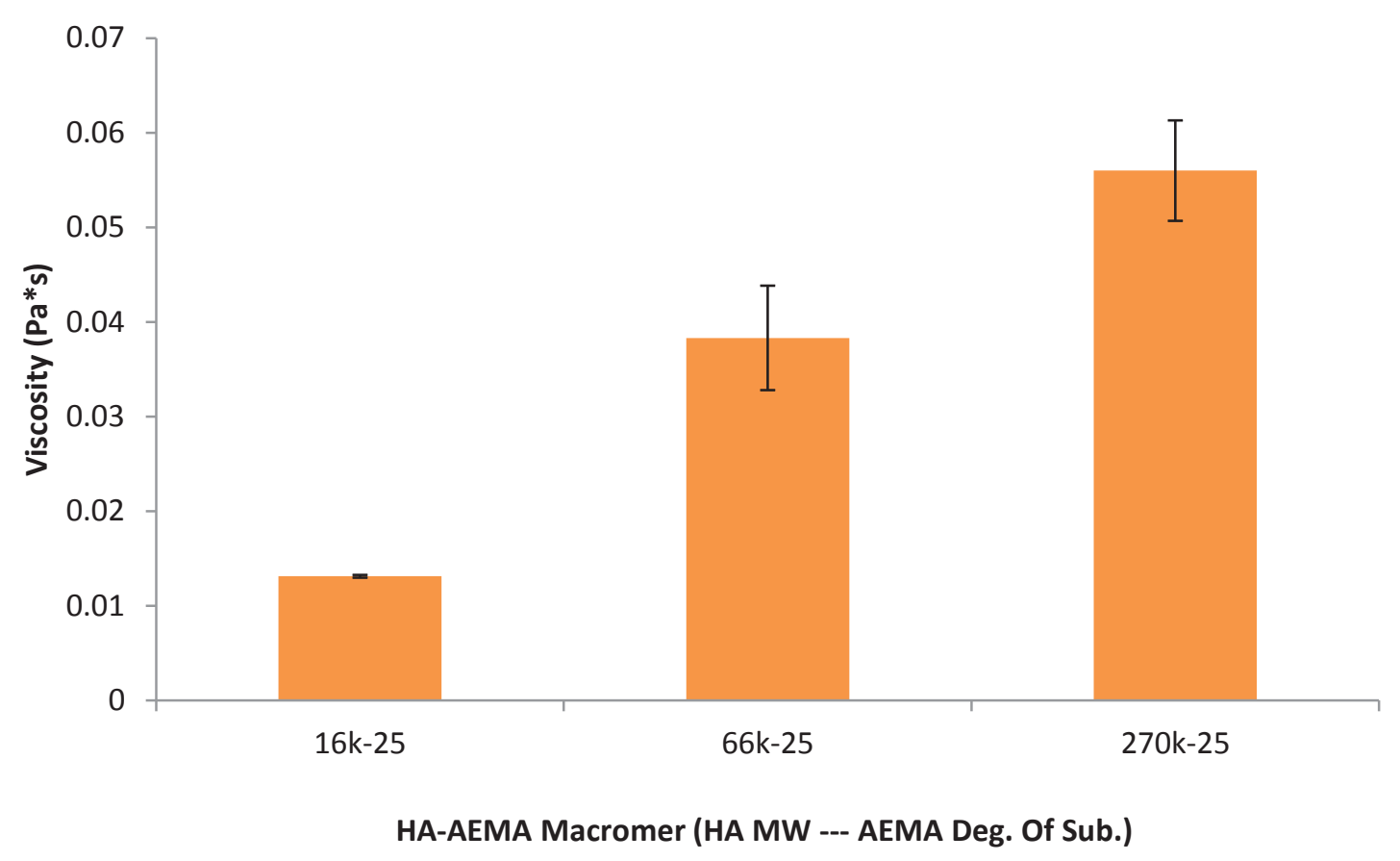

Figure 4-4. Viscosity of HA-AEMA-16k-25, HA-AEMA-66k-25, and HA-AEMA270k-25 macromer solutions.

Increased molecular weight of the HA component in the macromer directly affects the viscosity of the macromer solution. There were significant differences between $16 \mathrm{k}-25$ $(0.0131 \pm 0.0001 \mathrm{~Pa}-\mathrm{s})$ and $66 \mathrm{k}-25(0.0383 \pm 0.005 \mathrm{~Pa}-\mathrm{s})$ as well as between $66 \mathrm{k}-25$ and $270 \mathrm{k}-25(0.0560 \pm 0.005 \mathrm{~Pa}-\mathrm{s})$. P-values calculated from comparisons of 16k-25:66k-25 and $66 \mathrm{k}-25: 270 \mathrm{k}-25$ were $\mathrm{p}<0.01$ and $\mathrm{p}<0.05$, respectively. All viscosities were measured at $3 \%$ concentration, and the error bars represent standard deviation. $\mathrm{N}=3$. 
Table 4-2. Hydrogel formation of HA-AEMA macromers via UV polymerization.

\begin{tabular}{ccccccc}
\hline Macromer & \multicolumn{6}{c}{ Concentration of Macromer in Photo Initiator Solution (wt\%) } \\
\cline { 2 - 6 } & $\mathbf{0 . 1}$ & $\mathbf{0 . 5}$ & $\mathbf{1}$ & $\mathbf{2}$ & $\mathbf{3}$ & $\mathbf{5}$ \\
\hline $\begin{array}{c}\text { HA-AEMA- } \\
\text { 6.4k-25 }\end{array}$ & Failure & Failure & Failure & Failure & Failure & Failure \\
$\begin{array}{c}\text { HA-AEMA- } \\
\text { 16k-25 }\end{array}$ & Failure & Failure & Failure & Hydrogel & Hydrogel & Hydrogel \\
$\begin{array}{c}\text { HA-AEMA- } \\
\text { 18k-25 }\end{array}$ & Failure & Failure & Failure & Hydrogel & Hydrogel & Hydrogel \\
$\begin{array}{c}\text { HA-AEMA- } \\
\text { 66k-12.5 }\end{array}$ & Failure & Failure & Failure & Hydrogel & Hydrogel & Hydrogel \\
$\begin{array}{c}\text { HA-AEMA- } \\
\text { 66k-25 }\end{array}$ & Failure & Failure & Failure & Hydrogel & Hydrogel & Hydrogel \\
$\begin{array}{c}\text { HA-AEMA- } \\
\text { 270k-25 }\end{array}$ & Failure & Failure & Failure & Hydrogel & Hydrogel & Hydrogel \\
\hline
\end{tabular}




\section{Swelling of Hydrogels in PBS}

For HA-AEMA-66k-12.5 hydrogels, swelling results in a very flat, translucent hydrogel that is much more fluid than solid, and they became too fragile to handle at the 4 hour mark. However, all other hydrogel formations, which had a degree of substitution of $25 \%$, swelled to a cylindrical shape (the same shape as the Teflon container in which they were photopolymerized) and were translucent yet still visible. The difference in shape of HA-AEMA-66k-12.5 versus the other hydrogels can be seen in Figure 4-5.

Swelling ratios of were calculated for both HA-AEMA-16k-25 and HA-AEMA66k-25 hydrogels and compared after 3 days of swelling time in PBS. After swelling 5 hydrogels for each hydrogel type, the swelling ratio for $66 \mathrm{k}-25(78.4 \pm 5.2)$ was found to be significantly higher than $16 \mathrm{k}-25(50.6 \pm 7.6)(\mathrm{p}<0.01)$. The increase in swelling ratio for the 66k-25 hydrogels over the 16k-25 hydrogels indicates that swelling ratio increases as the molecular weight of HA increases, which allows a larger ingress of water to enter and be trapped in the hydrogel.

\section{Storage Modulus Measurement}

Comparisons for storage modulus were made between HA-AEMA-16k-25, HAAEMA-66k-25, and HA-AEMA-270k-25 hydrogels at a concentration of $3 \mathrm{wt} \%$. Figure 4-6 shows the results of the storage modulus measurements. The storage modulus of 66k25 hydrogels $(465.5 \pm 16.7 \mathrm{~Pa})$ were significantly higher than $16 \mathrm{k}-25$ hydrogels $(154.0 \pm$ $96.4 \mathrm{~Pa})(\mathrm{p}<0.05)$, but the difference between $66 \mathrm{k}-25$ and $270 \mathrm{k}-25(539.5 \pm 70.8 \mathrm{~Pa})$ was not significant $(\mathrm{p}>0.05)$. Based on the results of these t-test comparisons, there may be a threshold for HA molecular weight that, after going beyond this point, does not increase the effective storage modulus of the hydrogel. These are the same findings as found in the viscosity experiments, so a measurement of the viscosity of the macromer solution may be an indicator of the storage modulus following UV polymerization. This makes sense: since viscosity is a measure of internal friction in the fluid, a hydrogel formed from higher viscosity macromer solution will retain that same restriction to internal flow as a viscoelastic material. When a force is applied to the hydrogel, the restriction to flow of the viscous portion of the material allows more energy to be conserved rather than lost, thus increasing the storage modulus of the hydrogel.

Comparisons for storage modulus were made between HA-AEMA-66k-25 and HA-AEMA-66k-12.5 hydrogels at a concentration of 3\%. Figure 4-6 shows the results. T-test statistical analysis resulted in a vastly significant difference between $66 \mathrm{k}-12.5$ $(13.8 \pm 4.8 \mathrm{~Pa})$ and $66 \mathrm{k}-25(465.5 \pm 16.7 \mathrm{~Pa})(\mathrm{p}<0.001)$. This indicates that the degree of substitution of AEMA is a critical component to the mechanical integrity of the hydrogel. A substitution degree of $12.5 \%$ may be near the critical point for which a hydrogel would not even form (specifically for a molecular weight of 66k for HA), which could explain for the drastic drop in the storage modulus. An increase to the substitution degree, just as the increase in molecular weight showed, restricts the internal flow of the viscous portion of the hydrogel. Therefore, when forces are acted upon the hydrogel, there is less energy lost to the viscous portion, thus increasing the storage modulus. It seems that degree of 

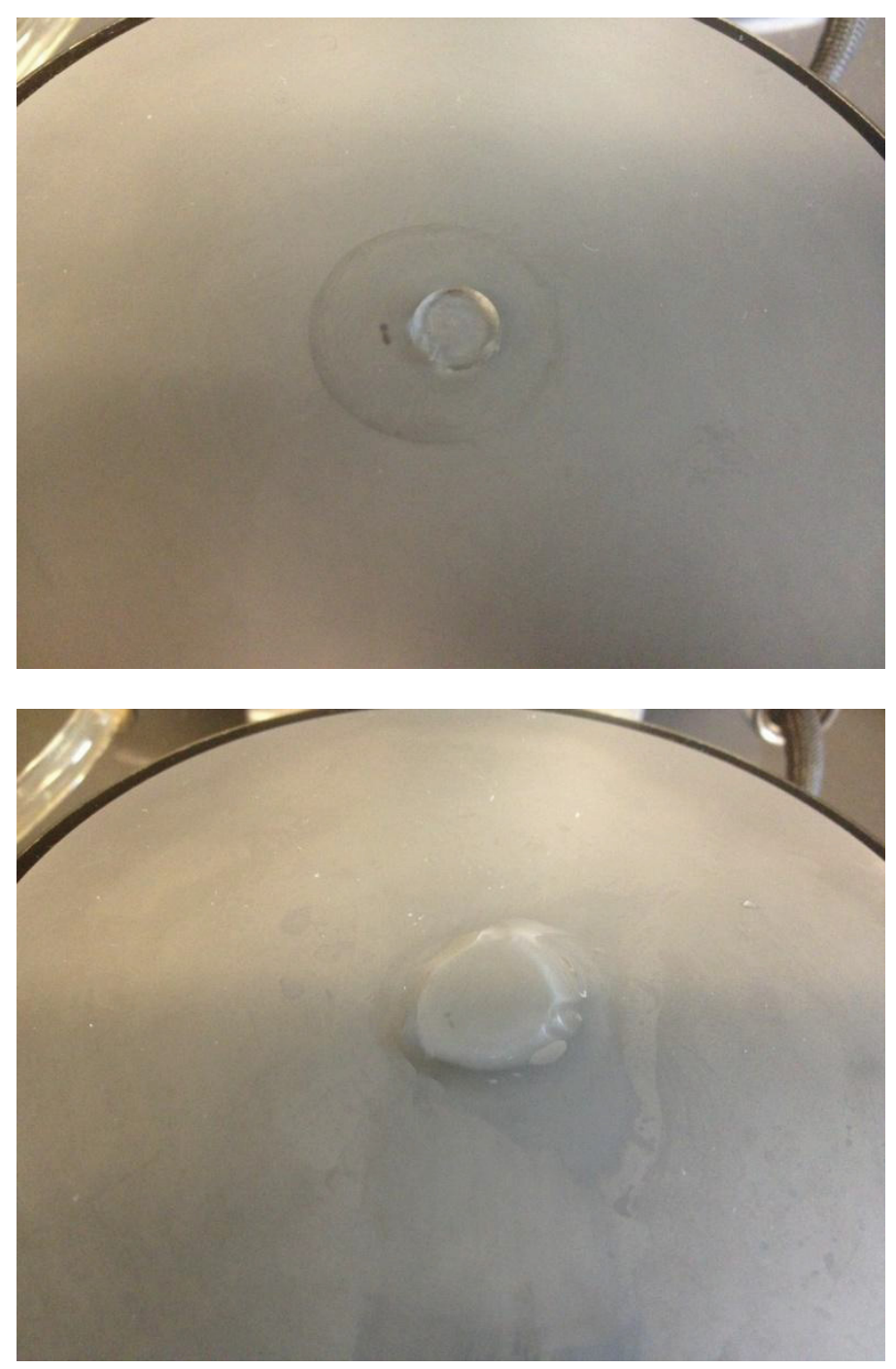

Figure 4-5. Difference in swollen hydrogels based on AEMA degree of substitution.

Top: HA-AEMA-66k-25 hydrogel. Bottom: HA-AEMA-66k-12.5 hydrogel. The lesser degree of substitution results in a flattened hydrogel that loses the shape of the container it was formed inside. HA-AEMA-66k-25 (width $=8 \mathrm{~mm}$ ) has a more well-defined shape and is much less viscous than HA-AEMA-66k-12.5 (width $=13 \mathrm{~mm}$ ). 


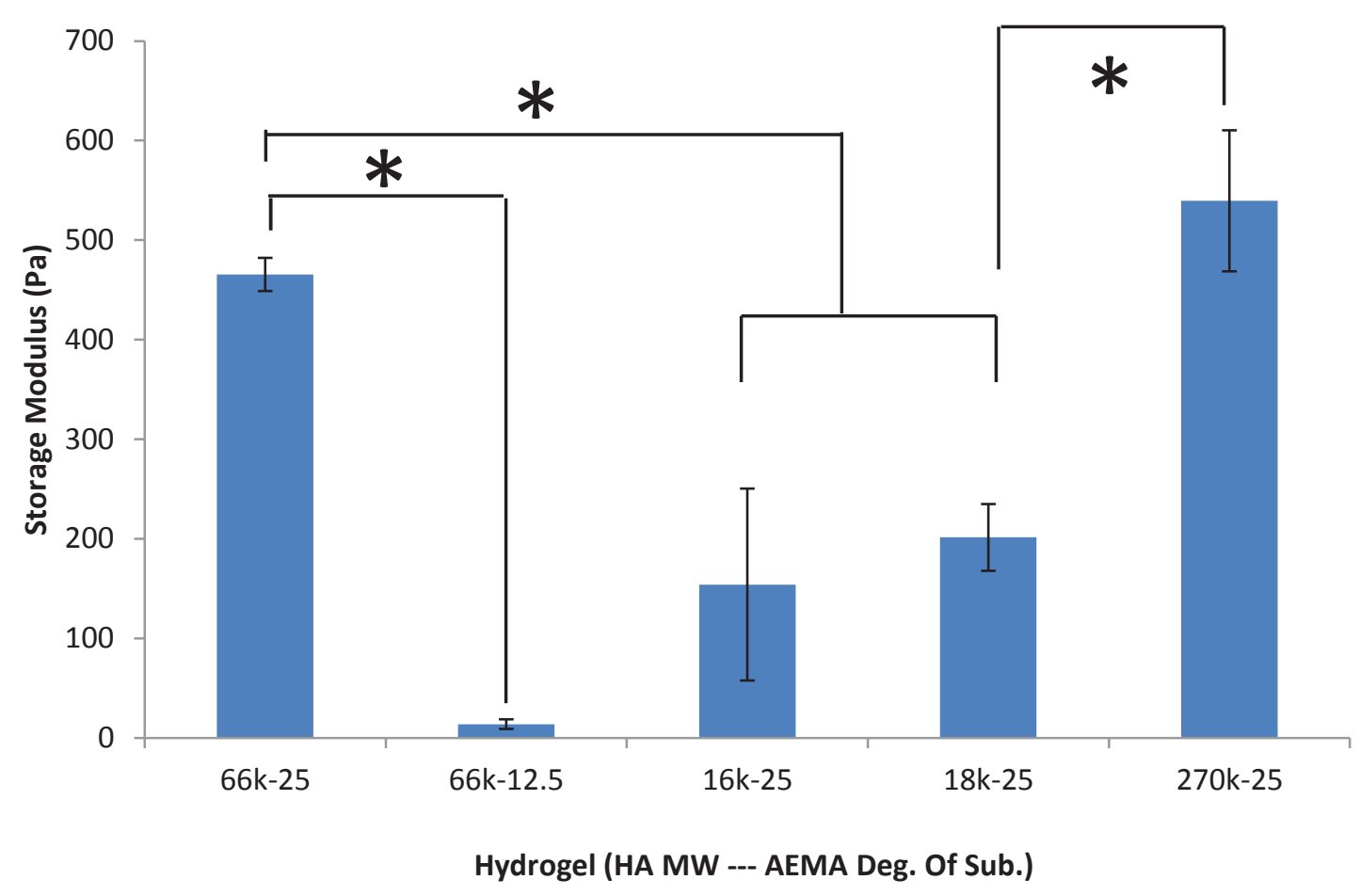

Figure 4-6. Storage modulus comparisons based on MW of HA, AEMA degree of substitution.

As both the molecular weight of HA and the AEMA degree of substitution increases, the storage modulus tends to increase, especially in comparisons between 66k-25 and hydrogels lower in both molecular weight and degree of substitution. The degree of substitution seems to have a stronger effect, evidenced by $66 \mathrm{k}-12.5$ being weaker than 16k-25. 16k-25 (154.0 $\pm 96.4 \mathrm{~Pa})$ and 18k-25 $(201.3 \pm 33.5 \mathrm{~Pa})$ showed similar storage properties. Though $270 \mathrm{k}-25(539.5 \pm 70.8 \mathrm{~Pa})$ hydrogels had a higher average storage modulus than $66 \mathrm{k}-25$ (465.5 $\pm 16.7 \mathrm{~Pa})$, this difference was not significant. Error bars represent standard deviations and $\mathrm{N}=3$ for all hydrogel configurations. The symbol "**" indicates a significant difference $(\mathrm{p}<0.05)$. 
substitution may be a bigger factor in its effect on the storage modulus over the molecular weight of the HA, just as it was the limiting factor in the solubility test for the macromers.

The storage modulus of HA-AEMA-16k-25 was compared at concentrations of $3 \%$ and $5 \%$. The storage modulus for $5 \%$ was significantly higher than the $3 \%$ concentration, measured at $(850 \pm 200 \mathrm{~Pa})$ and $(370 \pm 170 \mathrm{~Pa})$, respectively $(\mathrm{p}<.05)$.

\section{Compression Strength Measurement}

The profile of the stress/strain curve can be seen in Figure 4-7, and the results of the compression strength measurements can be seen in Figure 4-8. For each swelling time point, the compression profiles are all similar and do not significantly change over the period of 28 days. As the hydrogel is initially compressed, the strain on the hydrogels increased drastically with very little stress application (slope is very close to zero), and this is most likely due to water being expelled from the hydrogel. Once the hydrogels were compressed $60 \%$ to $80 \%$ of their original thickness, the slope of the stress/strain curve increased significantly, and the relationship of the curve became linear, forming the portion of the profile where the modulus was analyzed. For some of the hydrogel compression measurements, this linear region was followed by a pronounced flattening of the compression profile, most likely due to rupture of the hydrogel and the DMA pushing the fractured pieces outward. Some compression profiles showed no flattening region and the DMA automatically stopped measurement at the moment of fracture. Some compression profiles that had flattening regions following the modulus measurement section had a drastic increase following the flattening out region; with a modulus measuring in the gigapascals (GPa), this is most likely due to the machine compressing on itself rather than it being a measurement of the hydrogel strength.

In regard to the modulus measurements of the compressed HA-AEMA-18k-25 hydrogels, the compressive strength decreases as the swelling time increases. The dropoff in compressive strength is most pronounced between immediate compression following UV polymerization and swelling for 24 hours $(p<.005)$. The drop in compressive strength is also significant between 1 and 3 days of swelling time $(p<.01)$, but there isn't another significant drop in compressive strength until the swelling time reaches 21 days $(\mathrm{p}<.05)$. In addition, the compressive strength of HA-AEMA-270k-25 hydrogels is significantly higher than HA-AEMA-18k-25 hydrogels $(\mathrm{p}<0.001)$.

\section{Cell Survival and Encapsulation Studies}

\section{Cytotoxicity of HA-AEMA Macromer to DPSCs}

The viability of dental pulp stem cells to macromers HA-AEMA-18k-25 and HAAEMA-270k-25 were similar to the control for all 3 concentrations for both cases, seen 


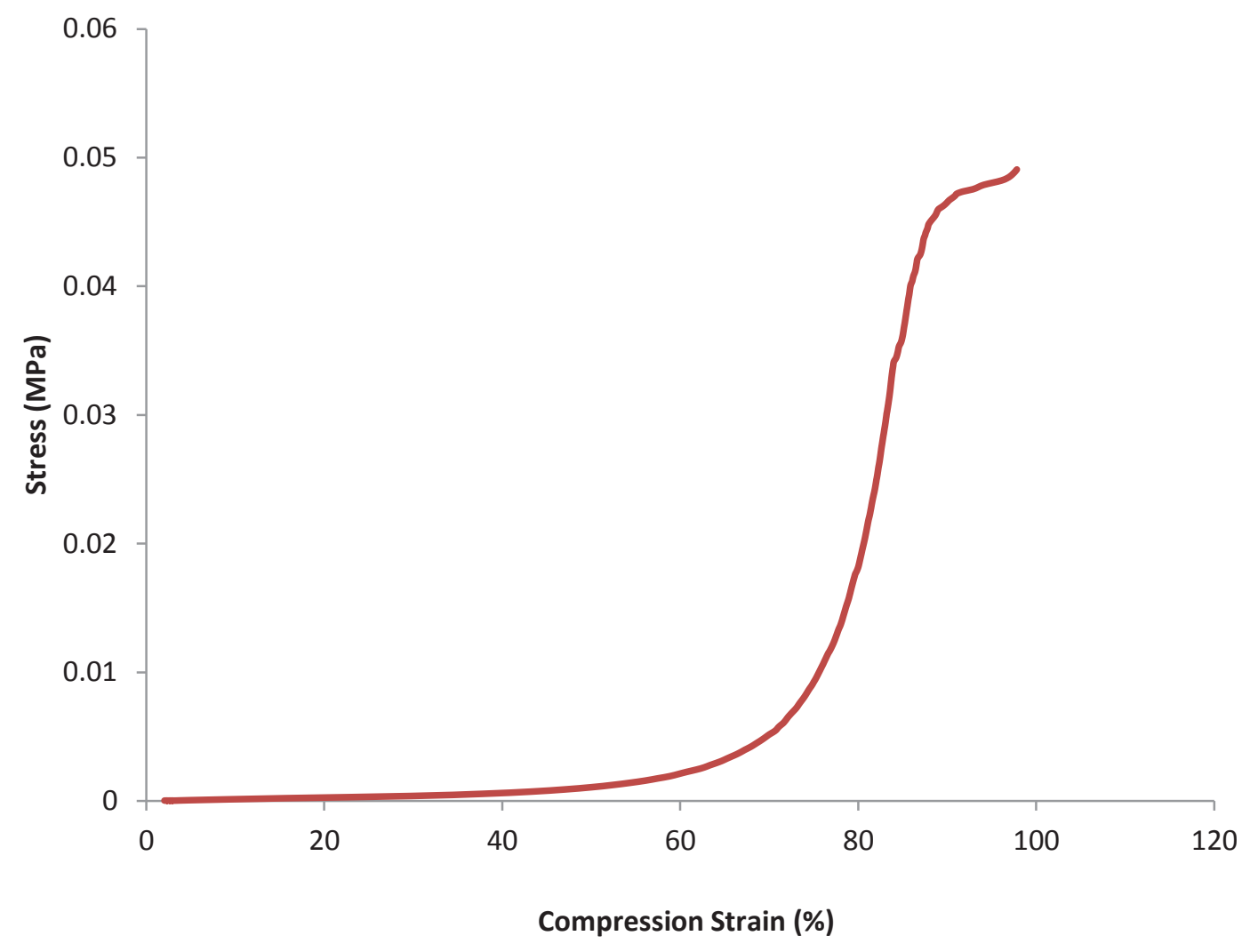

Figure 4-7. Profile of stress/strain curve for HA-AEMA hydrogel compression. Hydrogels are easily compressed as force is initially applied due, most likely, to water expulsion from within the hydrogel network. Between $60 \%$ and $80 \%$ strain, curves for all compressed hydrogels rise sharply into a linear region at which the elastic modulus was measured. Following the linear region, the curve will plateau, and this is most likely due to rupture of the hydrogel (all compressed hydrogels were destroyed as a result of the experiments). The curve shown above is a HA-AEMA-18k-25 hydrogel that was compressed immediately following polymerization. 


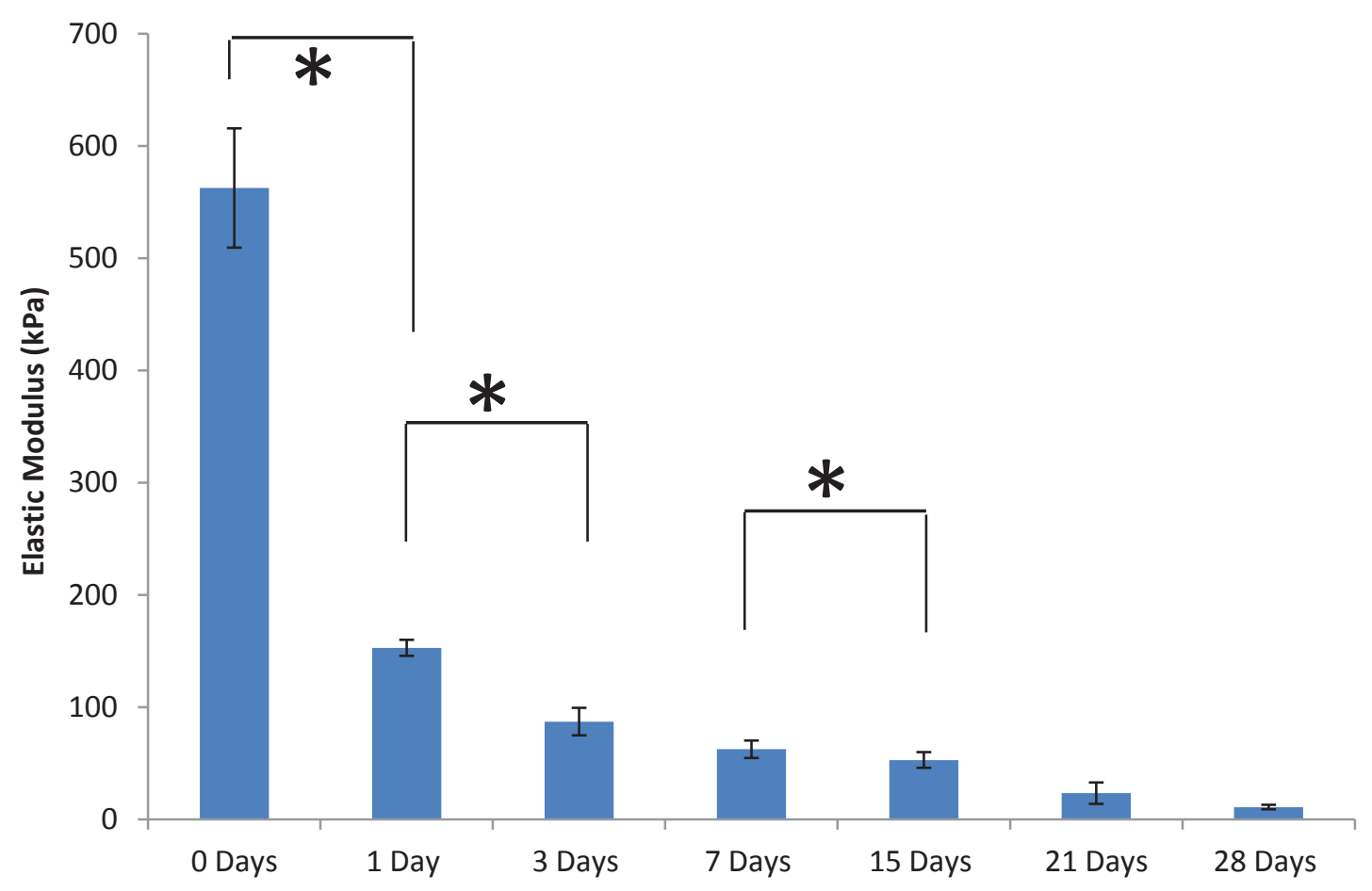

Figure 4-8. Compressive moduli of HA-AEMA-18k-25 hydrogels over time. The elastic moduli of hydrogels decreases as the swelling time increases. The most pronounced drop in compressive strength is observed between immediate compression following UV polymerization ( 0 days) and 1 day of swelling time $(\mathrm{p}<0.005)$. Significant differences are also found between 1 day and 3 days ( $<<0.01), 3$ days and 21 days $(\mathrm{p}<0.005), 7$ days and 21 days $(\mathrm{p}<0.05)$, and 15 days and 21 days $(\mathrm{p}<0.05)$. Immediate compression following UV polymerization for HA-AEMA-270k-25 hydrogels show a significant difference from HA-AEMA-18k-25 hydrogels ( $\mathrm{p}<0.001$, not shown). Errors represent standard deviation, and $\mathrm{N}=3$ for each time point. The symbol “*” indicates a significant difference $(\mathrm{p}<0.05)$. 
in Figure 4-9. Statistical analysis shows that there are no significant differences between any group when compared to the control or to any other group ( $\mathrm{p}>.05$ for all). An exposure time of 30 minutes, though a short duration, is considerably more time than the cells would be exposed to the macromer solution before undergoing UV polymerization. The double bond present on AEMA is what is considered toxic to the cells, so an exposure to this feature would be best kept at a minimum. Cells would only be exposed, at most, for a few minutes before immediately being put under UV for formation of the hydrogel. Based on the results, exposing DPSCs to HA-AEMA macromer for 30 minutes does not have a significant impact on the viability of the cells when compared to cells that are not exposed to HA-AEMA macromer.

\section{DPSC Encapsulation in Hydrogel}

Images of DPSCs encapsulated inside HA-AEMA hydrogel networks, both HAAEMA-18k-25 and HA-AEMA-270k-25 for a period of 24 hours and stained for live cells can be seen in Figure 4-10. After soaking the hydrogels in the calcein AM solution for 30 minutes, the obtained images confirmed successful encapsulation and survival of cells within the hydrogel network. Images were obtained both in fluorescence and light mode in order to overlap the coinciding images for comparison. Results of overlapping the images shows LIVE staining most concentrated within the encapsulated cells, indicating successful survival. Cells were successfully stained within the hydrogel both at the boundary and in the middle of the hydrogel, therefore the hydrogel is permeable and allows cells to interact with the external environment even if cells are encapsulated farther from the boundary than other cells. In addition, the images show no apparent migration of cells outside the boundary of the hydrogel due to the lack of cell staining beyond these boundaries in the overlapped images, meaning that cells are most likely fixated during the UV polymerization step of hydrogel formation. Obtaining higher magnification images in conjunction with confocal microscopy imaging could be utilized for determining cell viability statistical data and cell morphology in the future. 


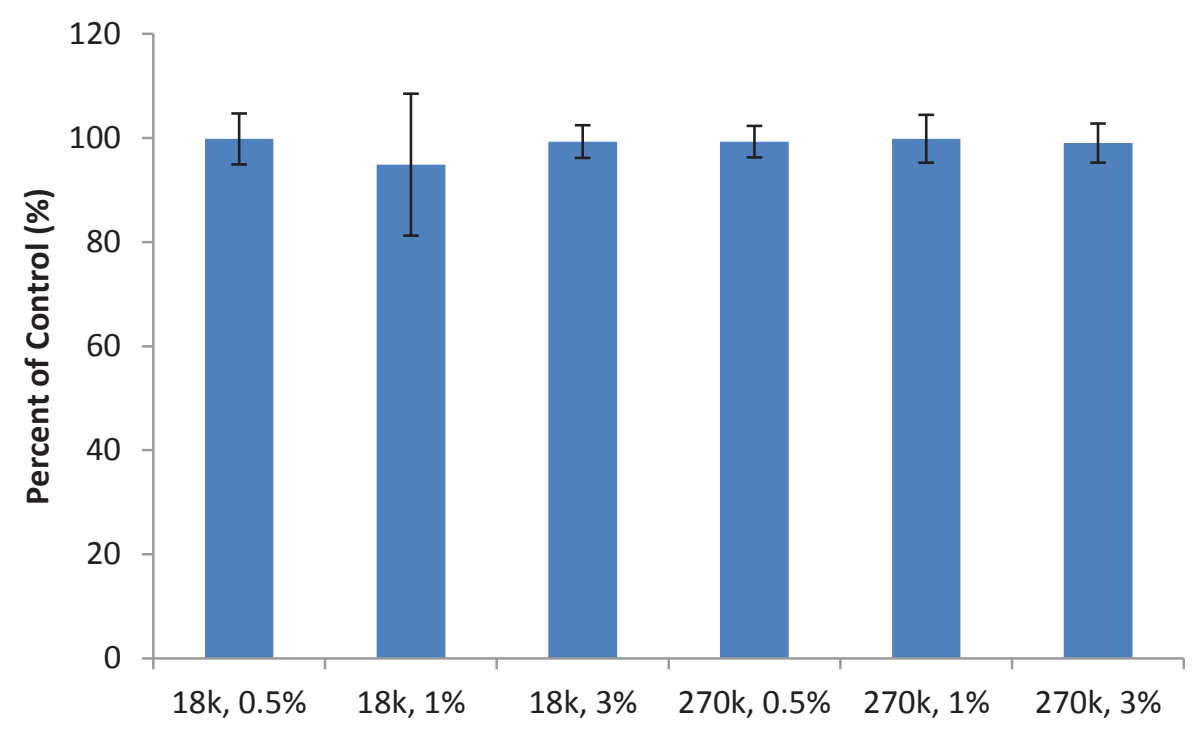

Figure 4-9. Cytotoxicity of HA-AEMA macromer to dental pulp stem cells. At each concentration, differences between the control and the macromer solution were not significant; therefore, cell viability was a success for every macromer tested. 30 minutes of exposure time is plenty considering the cell / macromer mixture would immediately be exposed to UV for hydrogel formation. 


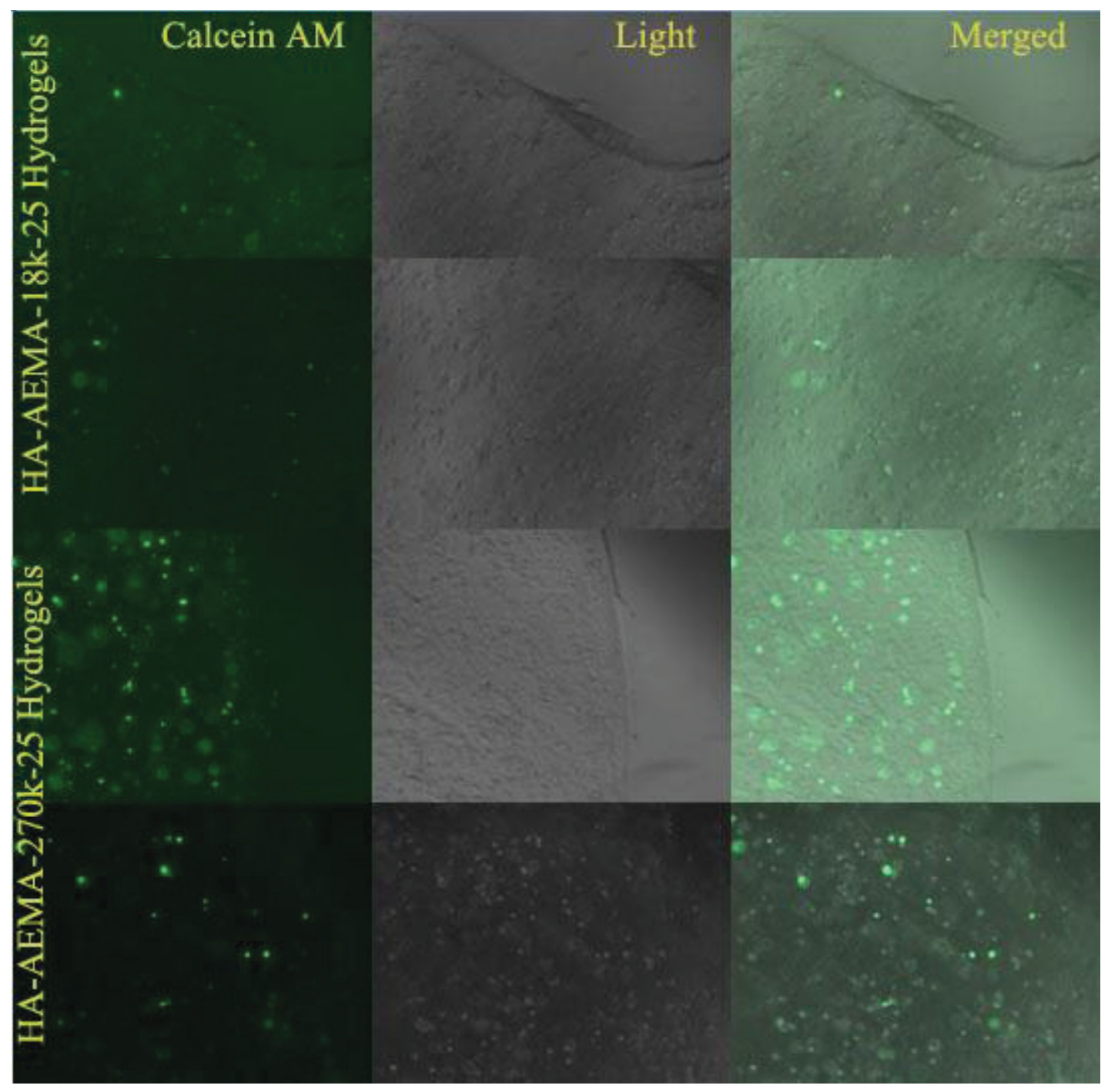

Figure 4-10. LIVE cell staining of encapsulated dental pulp stem cells in HAAEMA hydrogels.

Hydrogels containing DPSCs were imaged for calcein AM staining (column 1) and in light mode (column 2); these images were merged following image capture (column 3). DPSCs were successfully encapsulated and survived 24 incubation in growth medium for both HA-AEMA-18k-25 (rows 1,2) and HA-AEMA-270k-25 (rows 3,4). Cells did not permeate out of the scaffold after incubation as seen at the boundary of the hydrogels (rows 1,3 ). Images were taken at $4 \mathrm{X}$ magnification. 


\section{CHAPTER 5. DISCUSSION}

Synthesis of HA-AEMA macromer can vary widely in two different categories: the molecular weight of the HA component and the cross-linking density of the AEMA component. Though the only degree of substitution that was synthesized for this study was $25 \%$ and molecular weight of $\mathrm{HA}$ was $18 \mathrm{kDa}$ and $270 \mathrm{kDa}$, macromers differing in both of these factors have been synthesized and were tested for the mechanical and rheological studies (Bian, Hou et al. 2013, Guvendiren and Burdick 2013). Current technology is able to polymerize hyaluronan to a specific number of monomers in the chain, thus synthesis of HA-AEMA macromer can vary as widely as seen in nature in regards to the molecular weight of the HA component. The degree of substitution of AEMA during the conjugation step may also fluctuate, but results of both the solubility and UV polymerization tests suggest that this is the limiting factor between all possible synthesized macromers and those that are able to form a hydrogel; degrees of substitution around $12.5 \%$ do form hydrogels, but the hydrogel begins to degrade too quickly to be useful for application, yet higher degrees of substitution limit the solubility of the macromer. The molecular weight of HA limits hydrogel formation on the lower end of the spectrum, as evidenced by the failure of HA-AEMA- $6.4 \mathrm{k}-25$ to form a hydrogel, but solubility of $270 \mathrm{kDa}$ indicates the upper limit has not been reached. Hydrolysis is a concern during the synthesis process as it can lower the molecular weight of the HA chain. In some previous attempts at synthesis, dialysis was performed for periods of 3 to 5 days, yet each attempt failed to produce a macromer that would form a hydrogel. Though NMR results would show successful formation, and the macromer was soluble, hydrolysis from excessive dialysis broke down the HA backbone beyond the critical length needed to form an interweaving polymer network during UV polymerization. Once dialysis was lowered to 1 day, hydrogel formation became successful.

The rheological and mechanical properties of HA-AEMA macromers and hydrogels resulted in several direct relationships. Viscosity increases as the molecular weight of the HA component increases: this is because the longer HA chains of the macromer cause increased resistance to internal flow due to more interweaving and thus more sliding of polymer chains against each other. For the mechanical properties, storage modulus measurements also showed direct relationships for both cross-linking density and HA molecular weight. However, the drastic difference between the storage moduli of HA-AEMA-66k 12.5 and HA-AEMA-66k-25 suggests that the degree of substitution of AEMA may be more critical than molecular weight of HA in determining the overall shear strength of the hydrogel. Based on the HA component alone, increasing the molecular weight does increase the storage modulus, but the comparison between HAAEMA-66k-25 and HA-AEMA-270k-25 shows only a slight increase, suggesting that the direct relationship may correlate strongly at the lower end of the molecular weight spectrum. Testing macromers with HA molecular weights beyond $270 \mathrm{kDa}$ would help test this hypothesis. Increased concentration also showed an increase in storage modulus, and this also can be explained by the increased resistance to internal flow. By restricting the flow of the viscous component of the hydrogel, more energy can be stored in the hydrogel and the phase shift between the hydrogel action and the oscillatory force 
function is smaller, thus increasing the strength of the hydrogel's elastic portion. Measurement of the loss modulus was sporadic in comparison to the storage modulus, yet it increased for all hydrogels at the upper limits of the strain ramp during testing, thus all hydrogels displayed properties of shear thinning at strain percentages above the LVR.

Results of the compression data show a drastic reduction in strength as swelling time increases, and this is caused by hydrolysis of the glycosidic bond between the carbohydrate groups of the HA backbone. The breakdown of these bonds reduces the effective MW of the HA backbone, thus causing loss of strength, shape, and elasticity. The reduction in strength is most prevalent after 24 hours swelling, yet significant differences in strength are not seen again until 3 weeks of swelling time. Hydrogels with higher MW of HA have a significantly higher compression modulus immediately after UV polymerization, and these longer chains also result in a slower deterioration of the mechanical properties. As the hydrogel weakens, however, forces can more easily mold its shape, therefore making the scaffold more malleable in the body's environment.

DPSCs were able to survive the exposure to the macromer solution for 30 minutes at concentrations of $3 \%$ without significant difference. The amount of time the cells are exposed to the macromer is crucial, however. Experiments were conducted that exposed the macromer for a duration of 24 hours that saw significant differences in survival between the control and concentrations as small as $0.5 \% \mathrm{mg} / \mathrm{mL}$. Since the double bond on AEMA is considered toxic, limiting the exposure duration of cells to this feature is recommended. Exposure time of the cells to the macromer was kept to a minimum during encapsulation by immediately forming the hydrogel under UV following mixing the cells with the macromer solution. Immediate formation of the hydrogel minimizes the exposure of the cells to the AEMA double bond.

Based on the results of the encapsulation studies performed on the DPSCs into the HA-AEMA hydrogels, DPSCs are able to survive exposure to UV as well as incubation inside hydrogels swelling in growth medium for 24 hours. Calcein AM staining indicate that even after 24 hours of incubation, during which the degradation of the hydrogel by hydrolysis occurs, cells cannot migrate out of the scaffold, indicated by lack of fluorescence outside the scaffold. In addition, the ability of calcein AM to penetrate to the center of the hydrogel within 30 minutes also indicates that the hydrogel is an open system with the external environment. 


\section{CHAPTER 6. CONCLUSION}

Results of this study showed that HA-AEMA hydrogels can be formed with tunable properties for the purpose of encapsulating DPSC cells for potential tissue engineering purposes. By controlling the molecular weight of the HA component, the degree of substitution of AEMA, and the concentration of the macromer, the rheological and mechanical properties, specifically the viscosity, storage and loss moduli, and the compressive strength, can vary over a wide range of values. This is beneficial in terms of trying to match the mechanical properties of a biological scaffold to the in vivo environment. In addition, survival of the DPSCs during exposure to HA-AEMA macromer and encapsulation within the resulting hydrogels make these scaffolds a viable option for use as a biological scaffold for tissue engineering. The wound healing capabilities of HA further strengthen its choice as a foundation for a scaffold, and optimization of such a cell delivery system has the potential to be an effective treatment of tissue injury.

Future work would include expanding upon the finding of synthesis, mechanical and rheological properties of hydrogels, and cell culture work. More varieties of macromer can be synthesized, differing in both molecular weight of HA and the degree of substitution of AEMA. In regards to the molecular weight of HA, it can vary from the single-digit kiloDalton range all the way to millions of Daltons. The degree of substitution of AEMA to HA can also be controlled and varied during the conjugation step of synthesis. This will allow for a wider variety of studies to be performed in mechanical/rheological analysis and cell culture studies. As more varieties of macromer are synthesized, each new synthesis product can be tested for whether or not successful hydrogels may be created. As the limits of solubility, hydrogel formation through UV polymerization, and swelling are found, so can the limits of the mechanical and rheological properties of these hydrogels also be found. The full span of mechanical and rheological properties of the HA-AEMA hydrogel can be completely analyzed and recorded, specifically in regards to storage modulus and compression strength. In addition, hydrogels can be subjected to different oscillation frequencies to mark the changes made to their viscoelastic properties. Subjecting future macromer solutions and hydrogels to different temperature conditions may also result in changes to both mechanical and rheological properties; one study found that HA, when modified with collagen in a hydrogel form, increased the thermostability of the hydrogel for temperatures up to about $230^{\circ} \mathrm{C}$ (Pietrucha 2005). For future cell culture work, dental pulp stem cells, as well as other types of stem cells, may be grown and exposed to the full spectrum of HA-AEMA macromers that are able to form hydrogels to check for signs of cytotoxicity or whether the cells can survive the UV polymerization process. Particularly for encapsulation studies, changing the UV intensity or the duration of the UV exposure can help find the points at which significant differences are found between control and tested cells. Beyond cytotoxicity and encapsulation, future experiments need to focus on whether or not HA-AEMA hydrogels are able to assist in facilitating differentiation of stem cells for utilization in stem cell therapies of tissue engineering. 


\section{LIST OF REFERENCES}

Arthur, A., Rychkov G, Shi S, Koblar SA, Gronthos,S. (2008). Adult human dental pulp stem cells differentiate toward functionally active neurons under appropriate environmental cues. Stem Cells, 26(7), 1787-95.

Bae, M. S., Ohe, J. Y., Lee, J. B., Heo, D. N., Byun, W., Bae, H., Kwon, I. K. (2014). Photo-cured hyaluronic acid-based hydrogels containing growth and differentiation factor 5 (GDF-5) for bone tissue regeneration. Bone, 59, 189-198.

Bae, M. S., Yang, D. H., Lee, J. B., Heo, D. N., Kwon, Y. D., Youn, I. C., Kwon, I. K. (2011). Photo-cured hyaluronic acid-based hydrogels containing simvastatin as a bone tissue regeneration scaffold. Biomaterials, 32(32), 8161-8171.

Bencherif, S. A., Srinivasan, A., Horkay, F., Hollinger, J. O., Matyjaszewski, K., \& Washburn, N. R. (2008). Influence of the degree of methacrylation on hyaluronic acid hydrogels properties. Biomaterials, 29(12), 1739-1749.

Bian, L., Hou, C., Tous, E., Rai, R., Mauck, R. L., \& Burdick, J. A. (2013). The influence of hyaluronic acid hydrogel crosslinking density and macromolecular diffusivity on human MSC chondrogenesis and hypertrophy. Biomaterials, 34(2), 413-421.

Chang, C. Y., Chan, A. T., Armstrong, P. A., Luo, H. C., Higuchi, T., Strehin, I. A., Abraham, M. R. (2012). Hyaluronic acid-human blood hydrogels for stem cell transplantation. Biomaterials, 33(32), 8026-8033.

Chen, Q., Liang, S., \& Thouas, G. A. (2013). Elastomeric biomaterials for tissue engineering. Progress in Polymer Science, 38(3-4), 584-671.

Chen, W., \& Tong, Y. W. (2012). PHBV microspheres as neural tissue engineering scaffold support neuronal cell growth and axon-dendrite polarization. Acta Biomater, 8(2), 540-548.

Collins, M. N., \& Birkinshaw, C. (2013). Hyaluronic acid based scaffolds for tissue engineering--a review. Carbohydr Polym, 92(2), 1262-1279.

Du, J., Tan, E., Kim, H. J., Zhang, A., Bhattacharya, R., \& Yarema, K. J. (2014). Comparative evaluation of chitosan, cellulose acetate, and polyethersulfone nanofiber scaffolds for neural differentiation. Carbohydr Polym, 99, 483-490.

Fakhari, A., \& Berkland, C. (2013). Applications and emerging trends of hyaluronic acid in tissue engineering, as a dermal filler and in osteoarthritis treatment. Acta Biomater, 9(7), 7081-7092. 
Fraser, J.R. (1997). Hyaluronan: its nature, distribution, functions and turnover. Intern Med. 242(1), 27-33.

Gerecht, S., Burdick, J. A., Ferreira, L. S., Townsend, S. A., Langer, R., \& VunjakNovakovic, G. (2007). Hyaluronic acid hydrogel for controlled self-renewal and differentiation of human embryonic stem cells. Proc Natl Acad Sci U S A, 104(27), 11298-11303.

Gronthos, S., Mankani, M., Brahim, J., Robey, P.G., Shi, S. (2000). Postnatal Human Dental Pulp Stem Cells (DPSCs) in vitro and in vivo. Proc Natl Acad Sci U S A, 97(25), 13625-30.

Guvendiren, M., \& Burdick, J. A. (2013). Engineering synthetic hydrogel microenvironments to instruct stem cells. Curr Opin Biotechnol, 24(5), 841-846.

Hoffman, Matthew. "The Seven Most Common Sports Injuries." < http://www.webmd.com/men/features/seven-most-common-sports-injuries>.

Huang, G.T., Gronthos, S., Shi, S. (2009). Mesenchymal stem cells derived from dental tissues vs. those from other sources: their biology and role in regenerative medicine. J Dent Res, 88(9), 792-806

Katiyar, S., Pandit, J., Mondal, R. S., Mishra, A. K., Chuttani, K., Aqil, M., Sultana, Y. (2014). In situ gelling dorzolamide loaded chitosan nanoparticles for the treatment of glaucoma. Carbohydr Polym, 102, 117-124.

Kawashima, N. (2012). Characterisation of dental pulp stem cells: a new horizon for tissue regeneration? Arch Oral Biol, 57(11), 1439-1458.

Keane, T. J., \& Badylak, S. F. (2014). Biomaterials for tissue engineering applications. Semin Pediatr Surg, 23(3), 112-118.

Kim, I. L., Khetan, S., Baker, B. M., Chen, C. S., \& Burdick, J. A. (2013). Fibrous hyaluronic acid hydrogels that direct MSC chondrogenesis through mechanical and adhesive cues. Biomaterials, 34(22), 5571-5580.

Kim, Jennifer \& Smith, Joe (2009). Anterior Cruciate Ligament Injury (ACL). http://orthosurg.ucsf.edu/patient-care/divisions/sportsmedicine/conditions/knee/anterior-cruciate-ligament-injury-acl/

Leipzig, N. D., Wylie, R. G., Kim, H., \& Shoichet, M. S. (2011). Differentiation of neural stem cells in three-dimensional growth factor-immobilized chitosan hydrogel scaffolds. Biomaterials, 32(1), 57-64. 
Li, X., Katsanevakis, E., Liu, X., Zhang, N., \& Wen, X. (2012). Engineering neural stem cell fates with hydrogel design for central nervous system regeneration. Progress in Polymer Science, 37(8), 1105-1129.

Ma, C. Benjamin. “ACL Reconstruction.” Updated April 16, 2013. http://www.nlm.nih.gov/medlineplus/ency/article/007208.htm.

Mahoney, M. J., \& Anseth, K. S. (2006). Three-dimensional growth and function of neural tissue in degradable polyethylene glycol hydrogels. Biomaterials, 27(10), 2265-2274.

Meszar, Z., Felszeghy, S., Veress, G., Matesz, K., Szekely, G., \& Modis, L. (2008). Hyaluronan accumulates around differentiating neurons in spinal cord of chicken embryos. Brain Res Bull, 75(2-4), 414-418.

Nguyen, M. K., \& Alsberg, E. (2014). Bioactive factor delivery strategies from engineered polymer hydrogels for therapeutic medicine. Prog Polym Sci, 39(7), 1236-1265.

Oh, E. J., Park, K., Kim, K. S., Kim, J., Yang, J. A., Kong, J. H., Hahn, S. K. (2010). Target specific and long-acting delivery of protein, peptide, and nucleotide therapeutics using hyaluronic acid derivatives. J Control Release, 141(1), 2-12.

Pietrucha, K. (2005). Changes in denaturation and rheological properties of collagenhyaluronic acid scaffolds as a result of temperature dependencies. Int J Biol Macromol, 36(5), 299-304.

Price, R. D., Berry, M. G., \& Navsaria, H. A. (2007). Hyaluronic acid: the scientific and clinical evidence. J Plast Reconstr Aesthet Surg, 60(10), 1110-1119.

Schanté, C. E., Zuber, G., Herlin, C., \& Vandamme, T. F. (2011). Chemical modifications of hyaluronic acid for the synthesis of derivatives for a broad range of biomedical applications. Carbohydrate Polymers, 85(3), 469-489.

Seidlits, S. K., Khaing, Z. Z., Petersen, R. R., Nickels, J. D., Vanscoy, J. E., Shear, J. B., $\&$ Schmidt, C. E. (2010). The effects of hyaluronic acid hydrogels with tunable mechanical properties on neural progenitor cell differentiation. Biomaterials, 31(14), 3930-3940.

Seliktar, D. (2012). Designing cell-compatible hydrogels for biomedical applications. Science, 336(6085), 1124-1128.

Silva, A. K. A., Juenet, M., Meddahi-Pellé, A., \& Letourneur, D. (2014). Polysaccharidebased strategies for heart tissue engineering. Carbohydrate Polymers. 
Stern R. (2004). Hyaluronan catabolism: a new metabolic pathway. Eur J Cell Biol. 83(7), 317-25.

Tibbitt, M. W., \& Anseth, K. S. (2009). Hydrogels as extracellular matrix mimics for 3D cell culture. Biotechnol Bioeng, 103(4), 655-663.

Toh, W. S., Lim, T. C., Kurisawa, M., \& Spector, M. (2012). Modulation of mesenchymal stem cell chondrogenesis in a tunable hyaluronic acid hydrogel microenvironment. Biomaterials, 33(15), 3835-3845.

Vasi, A. M., Popa, M. I., Butnaru, M., Dodi, G., \& Verestiuc, L. (2014). Chemical functionalization of hyaluronic acid for drug delivery applications. Mater Sci Eng C Mater Biol Appl, 38, 177-185.

You, Z., X. Bi, X. Fan and Y. Wang (2012). A functional polymer designed for bone tissue engineering. Acta Biomaterialia, 8(2), 502-510.

Zhao, C., Tan, A., Pastorin, G., \& Ho, H. K. (2013). Nanomaterial scaffolds for stem cell proliferation and differentiation in tissue engineering. Biotechnol Adv, 31(5), 654668. 


\section{VITA}

Keegan Compton was born in 1988 in Murfreesboro, TN. He has an undergraduate degree from Vanderbilt University in Biomedical Engineering. After the acceptance of this thesis, he will have earned a Masters Degree in Biomedical Engineering from the University of Tennessee Health Science Center Biomedical Engineering Joint Program. 